TITLE:

\title{
Dynamic phase transitions in the anisotropic XY spin system in an oscillating magnetic field
}

$\operatorname{AUTHOR}(S)$ :

Yasui, T; Tutu, H; Yamamoto, M; Fujisaka, H

\section{CITATION:}

Yasui, T ... [et al]. Dynamic phase transitions in the anisotropic XY spin system in an oscillating magnetic field. Physical Review E 2002, 66(3): 036123.

ISSUE DATE:

2002-09

URL:

http://hdl.handle.net/2433/50326

RIGHT:

Copyright 2002 American Physical Society 
PHYSICAL REVIEW E 66, 036123 (2002)

\title{
Dynamic phase transitions in the anisotropic $X Y$ spin system in an oscillating magnetic field
}

\author{
Tomoaki Yasui, ${ }^{*}$ Hiroki Tutu ${ }^{\dagger}$ Mariko Yamamoto, ${ }^{\ddagger}$ and Hirokazu Fujisaka ${ }^{\S}$ \\ Department of Applied Analysis and Complex Dynamical Systems, Graduate School of Informatics, \\ Kyoto University, Kyoto 606-8501, Japan \\ (Received 19 April 2002; published 23 September 2002)
}

\begin{abstract}
The Ginzburg-Landau model for the anisotropic $X Y$ spin system in an oscillating magnetic field below the critical temperature $T_{c}, \dot{\psi}(\boldsymbol{r}, t)=\left(T_{c}-T\right) \psi-|\psi|^{2} \psi+\gamma \psi^{*}+\nabla^{2} \psi+h \cos (\Omega t)$ is both theoretically and numerically studied. Here $\psi$ is the complex order parameter and $\gamma$ stands for the real anisotropy parameter. It is numerically shown that the spatially uniform system shows various characteristic oscillations (dynamical phases), depending on the amplitude $h$ and the frequency $\Omega$ of the external field. As the control parameter, either $h$ or $\Omega$, is changed, there exist dynamical phase transitions (DPT), separating them. By making use of the mode expansion analysis, we obtain the phase diagrams, which turn out to be in a qualitative agreement with the numerically obtained ones. By carrying out the Landau expansion, the reduced equations of motion near the DPT are derived. Furthermore, taking into account the spatial variation of order parameters, we will derive the analytic expressions for domain walls, which are represented by the Néel and Bloch type walls, depending on the difference of the coexistence of phases.
\end{abstract}

DOI: 10.1103/PhysRevE.66.036123

PACS number(s): 64.60.-i, 05.45.-a, 75.60.-d

\section{INTRODUCTION}

After the first study of phase transition, such as dynamical behavior of a deterministic mean field equation of motion for a ferromagnet in an oscillating field [1,2], Monte Carlo studies of a kinetic Ising spin system under its critical temperature in a strong oscillating magnetic field have been carried out [3-7]. It was found that the system exhibits a symmetryrestoring oscillation (SRO) when the frequency $\Omega$ of the periodic external magnetic field is smaller than its critical value $\Omega_{c}$ determined by the temperature. This is because local spins can follow the slow variation of the external field. On the other hand, for a sufficiently high frequency field the spins cannot follow rapid change of the applied field, and ultimately the spins exhibit a symmetry-breaking oscillation (SBO).

In this connection the transition that is observed as the frequency is increased was called the dynamic phase transition (DPT). In particular, the analysis of the Monte Carlo simulation carried out for a two-dimensional kinetic Ising spin system below the critical temperature [4-7] suggests that the DPT belongs to the same universality class as that of the critical Ising spin system in thermal equilibrium. In fact, it was reported that in the SBO region the intensity of the deviation from the SRO increases in the power law form [3], and the probability density for the total magnetization averaged over one period of the oscillating field has a single peak structure in the SRO region and the two peak structure in the SBO region, as reported in Refs. [4-7]. It was also shown that various probabilistic cellullar automata with the symme-

\footnotetext{
*Electronic address: yasui@acs.i.kyoto-u.ac.jp

${ }^{\dagger}$ Electronic address: tutu@i.kyoto-u.ac.jp

${ }^{\ddagger}$ Present address: Hitachi Ltd., Central Research Laboratory 1280, Higashi-koigakubo Kokubunji-shi, Tokyo 185-8601, Japan; electronic address: m-yamamo@crl.hitachi.co.jp

${ }^{\S}$ Electronic address: fujisaka@i.kyoto-u.ac.jp
}

try the same as the Ising spin system belong to the same universality class as that of the equilibrium Ising spin system [8].

Recently, Fujisaka, Tutu, and Rikvold analyzed the Ginzburg-Landau equation in an oscillating external magnetic field,

$$
\dot{S}(\boldsymbol{r}, t)=\left(T_{c}-T\right) S-S^{3}+\nabla^{2} S+h \cos (\Omega t) \quad\left(T<T_{c}\right),
$$

to study the DPT from both the dynamical-theoretical and statistical-mechanical points of view [9], where $T$ is the temperature of the system and $T_{c}$ is its critical value. They found that the DPT is nothing but the bifurcation of the SRO which appears as either $\Omega$ is increased or $h$ is decreased. Furthermore, they carried out the Landau expansion around the unstable SRO including the thermal noise, and obtained the effective Hamiltonian the same as the Ginzburg-Landau free energy in the thermodynamic phase transition. This fact confirms that the DPT belongs to the same universality class as that of the Ising system in thermodynamic phase transition.

It is quite natural to ask whether or not such a phase transition is observed in other spin systems in an oscillating field. In Ref. [10], in fact, another type of DPT was reported in the Ginzburg-Landau equation with an oscillating external field corresponding to the $X Y$ spin system below its critical temperature. This fact suggests that there may exist rich varieties of DPT in other spin systems in an oscillating magnetic field. The aim of the present paper is to report on the analysis of the anisotropic spin system in an oscillating external magnetic field below the critical point. The model equation is the following Ginzburg-Landau type equation:

$$
\dot{\psi}(\boldsymbol{r}, t)=\left(T_{c}-T\right) \psi-|\psi|^{2} \psi+\gamma \psi^{*}+\nabla^{2} \psi+h \cos (\Omega t)
$$$$
\left(T<T_{c}\right),
$$ 
where $\psi$ is the complex order parameter, and $\gamma$ is a real anisotropy parameter. In the present model, the oscillating field is applied in the direction of the real component of $\psi$.

The present paper is organized as follows. In Sec. II, we will discuss the fundamental characteristics of the local dynamics of Eq. (1.2). It will be shown that the symmetry argument leads to the possibility of four types of oscillations, which suggests that there exist several DPTs separating them. In Sec. III, numerically integrating the local dynamics, we obtain the phase diagram for four dynamical phases for different values of the anisotropy parameter $\gamma$ with the Floquet analysis. In Sec. IV, we will carry out the analysis of the mode expansion to determine the DPT points and compare the results with those obtained in Sec. III. In Sec. V, to clarify the universality class of DPTs, we will derive the reduced dynamics, making use of the Landau expansion near DPTs, and its results are compared with exact results by numerical integration. In Sec. VI, considering the spatial inhomogeneity of spin variables, we will derive the analytic forms of domain walls connecting two stable oscillating states, and find that they are fundamentally the same as either the Néel or the Bloch walls. Concluding remarks are given in Sec. VII.

\section{GINZBURG-LANDAU MODEL AND SYMMETRY ARGUMENT}

After the rescaling

$$
\begin{gathered}
\psi \rightarrow\left(T_{c}-T\right)^{1 / 2} \psi, \quad t \rightarrow\left(T_{c}-T\right)^{-1} t, \quad \nabla \rightarrow\left(T_{c}-T\right)^{1 / 2} \nabla, \\
\gamma \rightarrow\left(T_{c}-T\right) \gamma, \quad h \rightarrow\left(T_{c}-T\right)^{3 / 2} h, \quad \Omega \rightarrow\left(T_{c}-T\right) \Omega,
\end{gathered}
$$

so that the dimension of temperature is unity, the anisotropic $X Y$ spin system Eq. (1.2) in a spatially uniform oscillating magnetic field with the amplitude $h$ and the frequency $\Omega$ is written as

$$
\dot{\psi}(\boldsymbol{r}, t)=\psi-|\psi|^{2} \psi+\gamma \psi^{*}+\nabla^{2} \psi+h \cos (\Omega t)=-\frac{\delta \mathcal{H}}{\delta \psi^{*}} .
$$

Here the Ginzburg-Landau Hamiltonian $\mathcal{H}$ is defined by

$$
\begin{aligned}
\mathcal{H}= & \int\left[-|\psi|^{2}+\frac{1}{2}\left(|\psi|^{2}\right)^{2}-\frac{\gamma}{2}\left(\psi^{2}+\psi^{* 2}\right)\right. \\
& \left.+|\nabla \psi|^{2}-h \cos (\Omega t)\left(\psi+\psi^{*}\right)\right] d \boldsymbol{r} .
\end{aligned}
$$

In the present paper, the thermal noise which is often added in the equation of motion is neglected. This is because we will mainly discuss the dynamics in a strong external magnetic field, where the deterministic nature of motion is not much affected by thermal noise. This model reduces to the Ising model [9] for $\gamma \rightarrow \infty$ and to the $X Y$ model [10] for $\gamma$ $=0$. The equations of motion for the components $X$ and $Y$ $(\psi=X+i Y)$ are rewritten as

$$
\begin{gathered}
\dot{X}(\boldsymbol{r}, t)=\left[1+\gamma-\left(X^{2}+Y^{2}\right)\right] X+\nabla^{2} X+h \cos (\Omega t)=-\frac{1}{2} \frac{\delta \mathcal{H}}{\delta X}, \\
\dot{Y}(\boldsymbol{r}, t)=\left[1-\gamma-\left(X^{2}+Y^{2}\right)\right] Y+\nabla^{2} Y=-\frac{1}{2} \frac{\delta \mathcal{H}}{\delta Y} .
\end{gathered}
$$

We first discuss the characteristics of the local spin dynamics without a spatial coupling term. The local dynamics is given by

$$
\dot{\psi}(t)=\psi-|\psi|^{2} \psi+\gamma \psi^{*}+h \cos (\Omega t) .
$$

Namely, by using the components, this is written as

$$
\begin{gathered}
\dot{X}(t)=\left[1+\gamma-\left(X^{2}+Y^{2}\right)\right] X+h \cos (\Omega t), \\
\dot{Y}(t)=\left[1-\gamma-\left(X^{2}+Y^{2}\right)\right] Y .
\end{gathered}
$$

For $h=0$ this set of equations of motion has the following linearly stable fixed points $\boldsymbol{X}^{s s}=\left(X^{s s}, Y^{s s}\right)$ :

$$
\begin{aligned}
& X^{s s}=( \pm \sqrt{1+\gamma}, 0) \quad(\gamma>0), \\
& \boldsymbol{X}^{s s}=\left(\cos \theta_{0}, \sin \theta_{0}\right),\left(\theta_{0}: \text { arbitrary }\right) \quad(\gamma=0), \\
& \boldsymbol{X}^{s s}=(0, \pm \sqrt{1-\gamma}) \quad(\gamma<0) .
\end{aligned}
$$

Without an external field, for the replacement $\gamma \rightarrow-\gamma$, the dynamics are equivalent since it only alters the role of the real and imaginary components. However, when $h \neq 0$, since the specific direction of the external field breaks the equivalence for the replacement $\gamma \rightarrow-\gamma$ followed by the exchange of the components, the dynamics for $\gamma>0$ and $\gamma<0$ are different.

The set of the equations of motion (2.6) and (2.7) involves two invariant properties for corresponding symmetry operations. The symmetry argument allows us to classify the dynamical behaviors of the system. Let us discuss the basic role of the symmetry in dynamics. First, if $\psi(t)=X(t)+i Y(t)$ is a solution of Eq. (2.5), then

$$
\widetilde{\psi}(t)=\psi^{*}(t)=X(t)-i Y(t)
$$

also satisfies Eq. (2.5). This reflects the property that Eq. (2.5) is invariant under the change of sign, $Y \rightarrow-Y$. One should note that these oscillations with $Y>0$ or $Y<0$ belong to separate regions in the phase space, because the sign of the $Y$-component cannot be changed in the dynamics. Furthermore, with the period $T(=2 \pi / \Omega)$ of the applied field, if $\psi(t)=X(t)+i Y(t)$ is a solution of Eq. (2.5), then

$$
\hat{\psi}(t)=-\psi^{*}\left(t+\frac{T}{2}\right)=-X\left(t+\frac{T}{2}\right)+i Y\left(t+\frac{T}{2}\right)
$$

is also the solution of Eq. (2.5). This reflects the property that Eq. (2.5) is invariant for the operations $t \rightarrow t+T / 2$ and $X \rightarrow$ $-X$.

The motion with the symmetry 

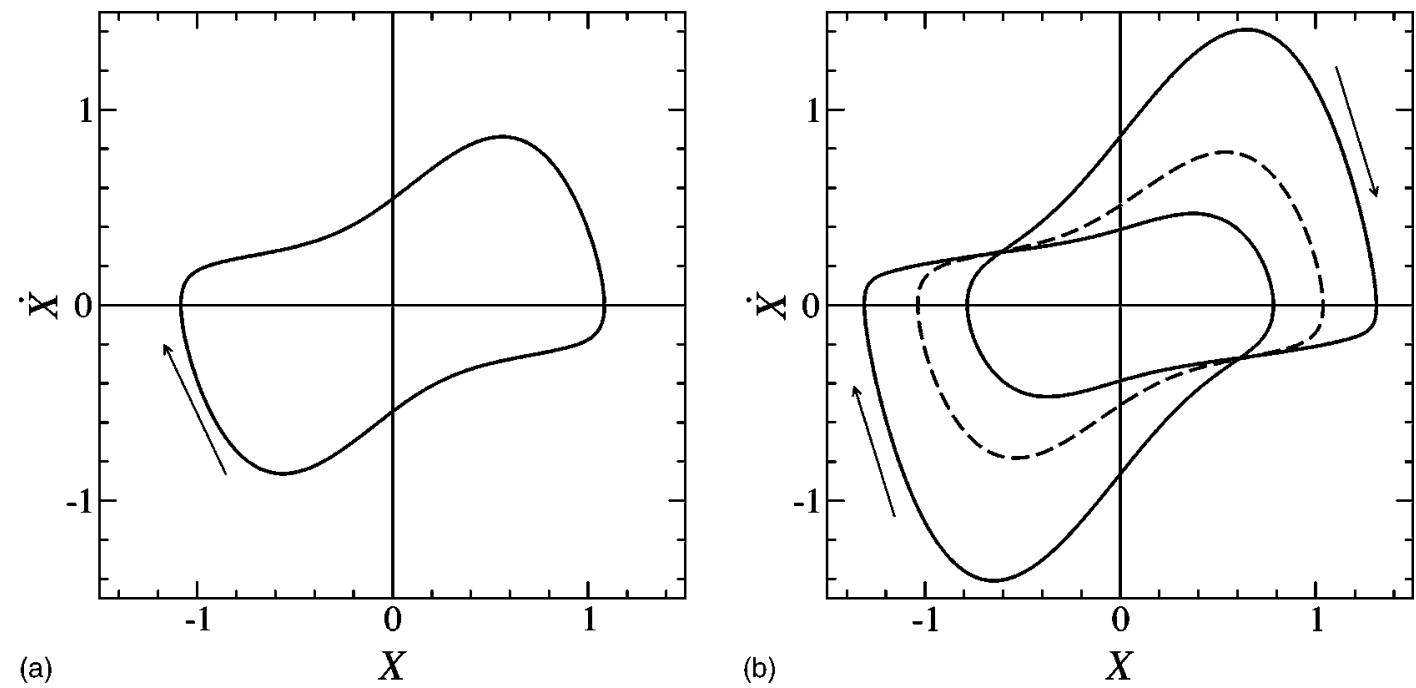

FIG. 1. Limit cycles in the $X-\dot{X}$ space denoted by solid lines respectively indicate typical trajectories of the Ising-SRO (a) and the Ising-SBO (b) phases. Solid and broken lines in (b) correspond to stable and unstable limit cycles, respectively. The broken line in (b) is the Ising-SRO. There are degenerate stable limit cycles in (b). Arrows show the movement of the phase points in the course of time. The parameters are $\gamma=0.55$ and $h=1.0$ are for (a) and (b), and, $\Omega=0.520$ for (a) and $\Omega=0.530$ for (b).

$$
\psi(t)=\psi^{*}(t), \text { i.e., } \quad Y(t)=0
$$

is called the Ising-type motion. An oscillation without this symmetry is called the XY-type motion. One can expect that the dynamics tends to exhibit the Ising-type motion for a large value of $|\gamma|$. On the other hand, if the motion has the symmetry

$$
\psi(t)=-\psi^{*}\left(t+\frac{T}{2}\right)
$$

its real part satisfies the condition

$$
\int_{0}^{T} X(t) e^{i l \Omega t} d t=0 \quad(l=0, \pm 2, \pm 4, \ldots) .
$$

Because of this particular symmetry, the motion with the symmetry Eq. (2.14) is called the SRO, and the motion without this symmetry is called the SBO.

As is expected, for a sufficiently large value of the anisotropy parameter $\gamma$, only the SBO is stable for a vanishing external field. As the magnitude of the external field is increased, the spin variable tends to synchronize the variation of the external field. Furthermore, as is explicitly shown in Appendix A, the local spin apparently shows the SBO for a weak external field. Therefore, one expects that if the SRO stably exists, it should be observed for sufficiently large values of the field amplitude. Furthermore, as the frequency of the external field is increased, it is expected that the spin cannot follow the variation of the external field.

The above simple argument suggests that depending on the amplitude and the frequency of the external field, the local dynamics (2.5) may exhibit four types of motions: Ising-SRO, Ising-SBO, $X Y$-SRO, and $X Y$-SBO. Therefore, we expect the existence of dynamic phase transitions among these characteristic oscillations.

\section{DYNAMIC PHASE TRANSITION AND PHASE DIAGRAM}

\section{A. Four dynamical phases}

In this section, carrying out numerical integration of Eq. (2.5), we show the existence of dynamic phase transitions as expected from the symmetry argument in the preceding section, and present phase diagrams in the space spanned by the frequency and the amplitude of the external field. The types of limit cycles are crucially affected by the magnitude of the anisotropy $\gamma$, and are thus classified with the value of $\gamma$. When $\gamma$ takes sufficiently a large positive value, it is expected that Eq. (2.5) is similar to the Ising model. Figure 1 shows the trajectories of limit cycles for a couple of parameter sets, $\gamma=0.55, h=1.0, \Omega=0.520$ [Fig. 1(a)], and $\Omega$ $=0.530$ [Fig. 1(b)]. These figures represent a couple of different Ising-type oscillations. Since they have a vanishing $Y$ component, the trajectories in the $X-\dot{X}$ space are shown. For $\Omega=0.520$, the Ising-SRO trajectory, which has the symmetries (2.13) and (2.14), is stable, which is shown in Fig. 1(a). On the other hand, for $\Omega=0.530$ the Ising-SBO trajectories with the symmetry (2.13) but without the symmetry (2.14) is stable, and the Ising-SRO trajectory is unstable. The IsingSBO trajectories are degenerate twofold. These two stable and one unstable trajectories are, respectively, represented by solid and broken curves in Fig. 1(b). As expected, the emergence of a couple of trajectories presented above confirms the existence of the DPT. Namely, there exists the transition point $\Omega_{1}\left(\Omega_{1} \approx 0.525\right)$, and the Ising-SRO and the IsingSBO phases are stable, respectively, for $\Omega<\Omega_{1}$, and for $\Omega$ $>\Omega_{1}$.

There is also an oscillation with a nonvanishing imaginary component of the order parameter for $\gamma<0$, which is referred to as the $X Y$-type oscillation. In the same way as the above, we compare the limit cycles for parameter values $\gamma$ $=-0.05, h=1.0, \Omega=0.317$, and $\Omega=0.337$. For $\Omega=0.317$ the Ising-SRO is stable, while for $\Omega=0.337$ the $X Y$-SRO is 


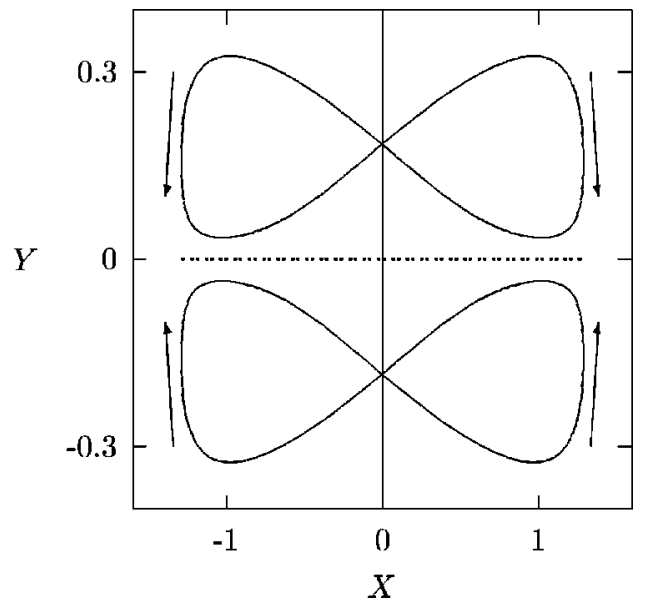

FIG. 2. Typical limit cycles in the $X Y$-SRO phase, where parameter values are $\gamma=-0.05, h=1.0$, and $\Omega=0.337$. The unstable, degenerate stable trajectories are indicated by the broken line and solid lines, respectively. Arrows show the movement of the phase points in the course of time.

stable. Therefore, there exists the dynamic phase transition at $\Omega=\Omega_{2}$. Namely, for $\Omega>\Omega_{2} \approx 0.327$ a couple of degenerate $X Y$-SRO trajectories are stable, while the Ising-SRO trajectory is unstable. Figure 2 shows the trajectories for $\Omega$ $=0.337$ in the $X Y$-SRO phase.

For the case where $\gamma$ is between values previously argued, we observe an oscillation without both types of the symmetries which is a proper motion in the original anisotropic $X Y$ model. Characteristic behaviors for the four different parameter values $\Omega=0.646, \Omega=0.833, \Omega=0.851$, and $\Omega=0.869$, where $\gamma=0.30$ and $h=1.0$ are kept common, are as follows. For $\Omega=0.646(\Omega=0.833)$, the Ising-SRO (the $X Y$-SRO) is stable. For $\Omega=0.851$, the $X Y$-SBO is stable, and for $\Omega$ $=0.869$, the Ising-SBO is stable. Thus, there are three dynamic phase transition points $\Omega_{2}, \Omega_{3}$, and $\Omega_{4}$ as $\Omega$ is changed, which separate the four dynamical phases. Figure 3 shows the limit cycles in the $X Y$-SBO phase corresponding

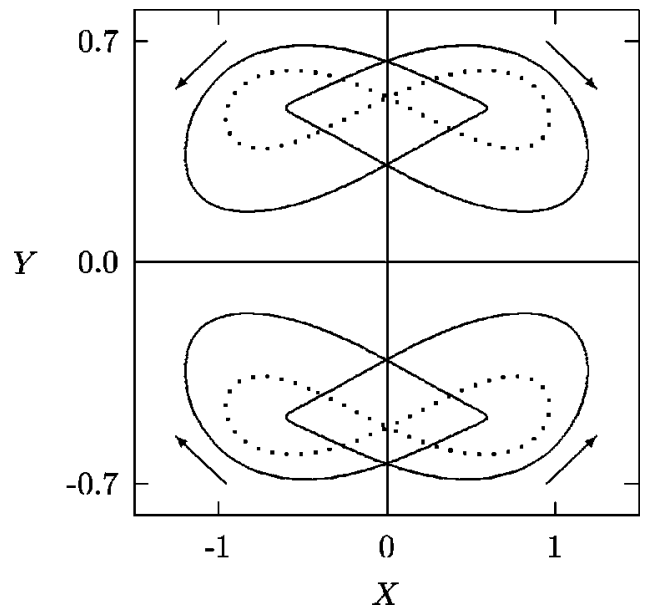

FIG. 3. Typical limit cycles in the $X Y$-SBO phase, where the parameter values are $\gamma=0.30, h=1.0$, and $\Omega=0.851$. The unstable and stable trajectories are indicated by the broken and solid lines in the $X-Y$ space, respectively. Arrows show the movement of the phase points in the course of time.

to the third parameter set. For $\Omega_{3}(\approx 0.843)<\Omega<\Omega_{4}$, a couple of the $X Y$-SRO trajectories are unstable (broken lines) and four $X Y$-SBO trajectories are stable (solid curves).

\section{B. Floquet analysis and phase diagrams}

Numerical values of the phase transition points can be obtained by utilizing the Floquet analysis. Let us denote the period-T solution of $\psi(t)$ by $\psi_{p}(t)$ and the deviation of $\psi(t)$ from $\psi_{p}(t)$ by $\delta \psi(t)$, i.e.,

$$
\psi(t)=\left(\begin{array}{c}
X(t) \\
Y(t)
\end{array}\right), \quad \psi_{p}(t)=\left(\begin{array}{c}
X_{p}(t) \\
Y_{p}(t)
\end{array}\right), \quad \delta \psi(t)=\psi(t)-\psi_{p}(t),
$$

The linearized equation for $\delta \psi(t)$ is written as

$$
\dot{\delta} \psi(t)=\hat{G}(t) \delta \psi(t)
$$

$$
\hat{G}(t)=\left(\begin{array}{cc}
1+\gamma-3 X_{p}(t)^{2}-Y_{p}(t)^{2} & -2 X_{p}(t) Y_{p}(t) \\
-2 X_{p}(t) Y_{p}(t) & 1-\gamma-X_{p}(t)^{2}-3 Y_{p}(t)^{2}
\end{array}\right),
$$

where the matrix $\hat{G}$ is the period- $T$ function, i.e., $\hat{G}(t+T)$ $=\hat{G}(t)$.

Using the matrix $\hat{U}(t)$ defined by $\hat{U}(t)=\hat{G}(t) \hat{U}(t)$ with the initial condition $\hat{U}(0)=\hat{1}$ ( $\hat{1}$ : the unit matrix), Eq. (3.2) is solved as $\delta \psi(t)=\hat{U}(t) \delta \psi(0)$. From the Floquet theory it can be shown that the solution $\hat{U}(t)$ can be written in the form [11]

$$
\hat{U}(t)=\hat{Q}(t) e^{t \hat{\Lambda}},
$$

where $\hat{Q}(t)$ is a period-T function, and $\hat{\Lambda}$ is the Floquet matrix defined by $\hat{U}(T)=e^{T \hat{\Lambda}}$. The stability of the periodic trajectory $\psi_{p}(t)$ is thus measured by the eigenvalues of the matrix $\hat{\Lambda}$. Namely, denoting the eigenvalues of $\hat{\Lambda}$ as $\lambda_{j}(j$ $=1,2)$, if $\operatorname{Re} \lambda_{j}<0 \quad(j=1,2)$, then the periodic trajectory $\psi_{p}(t)$ is stable for any small perturbation. Therefore, transition points, i.e., phase boundaries among different dynamical phases, are given by the vanishing points of the largest real part of the eigenvalues of $\hat{\Lambda}$. Figure 4 shows three typical behaviors of the Floquet exponents $\lambda$ for stable limit cycles as $\Omega$ is changed for three typical values of $\gamma$. By changing $\Omega$ 

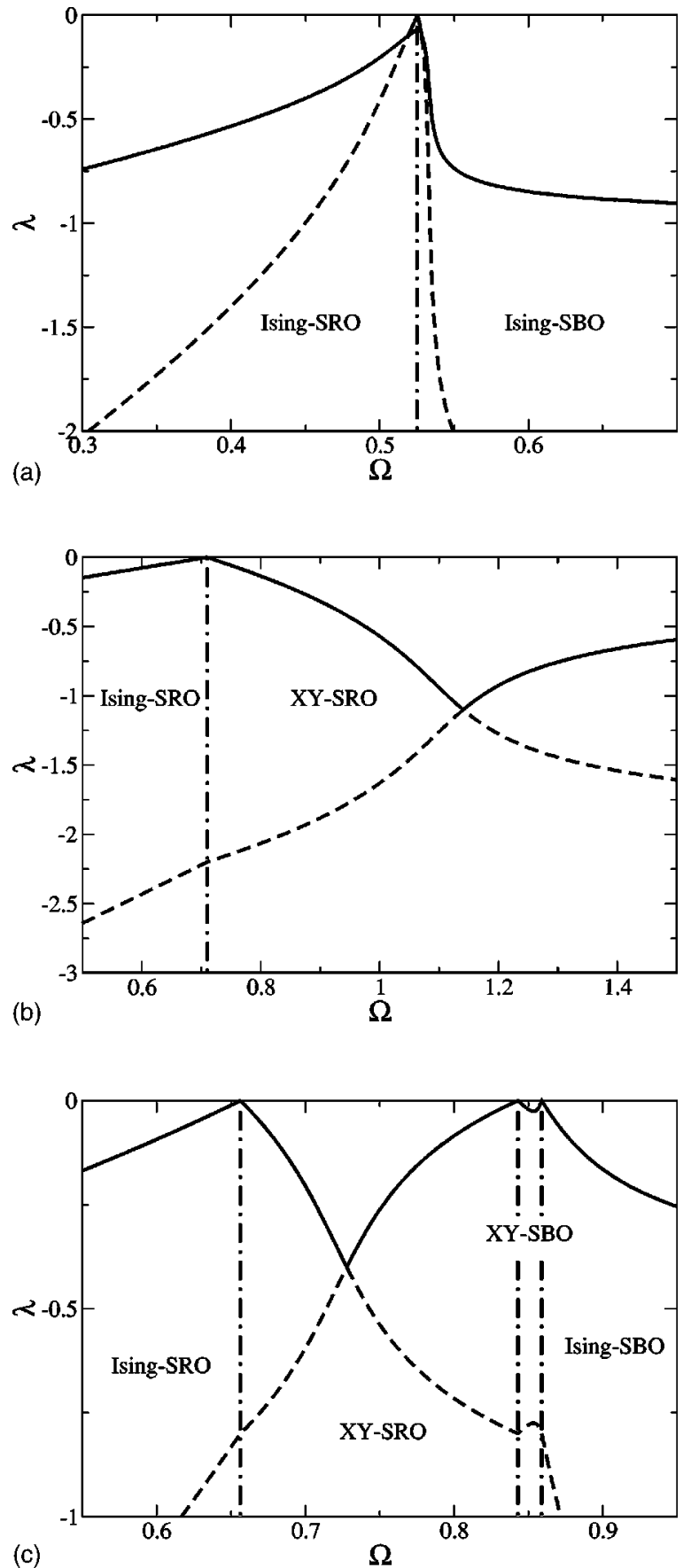

FIG. 4. Floquet exponents $\lambda$ for three typical values of $\gamma$ : (a) $\gamma=0.55, h=1.0$; (b) $\gamma=-0.05, h=1.5$; and (c) $\gamma=0.30, h=1.0$. Solid and broken lines correspond to the first and second Floquet exponents, respectively. The transition points separating different phases are indicated by dotted broken vertical lines, at which the largest Floquet exponent vanishes.

continuously, each of the Floquet exponents is measured for a stable limit cycle reached after the transient time is over. In the present system $\lambda$ 's are always real. Figure 4(a) represents the existence of the transition between the Ising-SRO and the Ising-SBO phases. The Ising-SRO phase exists for $\Omega<\Omega_{1}$ $(\approx 0.525)$, and the Ising-SBO phase exists for $\Omega>\Omega_{1}$. Figure 4 (b) shows the existence of the transition between the

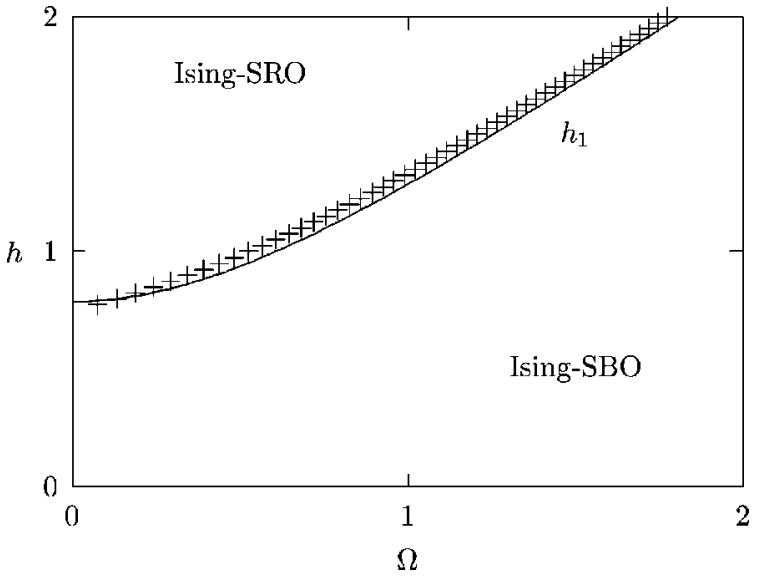

FIG. 5. Phase diagram for $\gamma=0.55$. The transition curve indicated by $h_{1} \equiv h_{1}(\Omega)$ separates the Ising-SRO and the Ising-SBO phases. Symbols and solid lines are obtained by the Floquet analysis and mode expansion analysis (see Sec. IV), respectively.

Ising-SRO and the $X Y$-SRO. The Ising-SRO and $X Y$-SRO phases are present for $\Omega$, respectively, below and above the transition point $\Omega_{2}(\approx 0.709)$. Figure $4(\mathrm{c})$ shows the case in which the successive transitions among the Ising-SRO, $X Y$-SRO, $X Y$-SBO, and Ising-SBO are present. The transition points are located at $\Omega_{2} \approx 0.656, \Omega_{3} \approx 0.843$, and $\Omega_{4}$ $\approx 0.859$.

As described above, using the Floquet exponents, we can determine the transition points among different dynamical phases, which enables us to make phase diagrams. In the remaining part of this section we show typical phase diagrams for three values of $\gamma$. For each $\gamma$, the transition curves in the $(\Omega, h)$ parameter space are surveyed by searching the vanishing points of the largest Floquet exponent. Figure 5 is the phase diagram for $\gamma=0.55$, where there are two phases: the Ising-SRO and the Ising-SBO phases. The transition curve is indicated by the symbols and the solid line, respectively, obtained by numerical calculation and the mode expansion analysis shown in the following section. As will be shown in the following section, in the region $\gamma \geqslant \frac{1}{2}$ there are only two phases, the Ising-SRO and Ising-SBO phases. This situation can be regarded as a strong anisotropic case, as is the same with the model studied in Ref. [9]. Figure 6 is the phase diagram for $\gamma=-0.05$. There are again two phases. However, in contrast to Fig. 5, they are Ising-SRO and $X Y$-SRO phases. As will be shown in the following section, in the region $\gamma \leqslant 0$ only the Ising-SRO and $X Y$-SRO phases exist, namely, all the region with $\gamma \leqslant 0$ is essentially the same phase behavior as that for the isotropic $X Y$ model studied in Ref. [10]. Figure 7 is the phase diagram for $\gamma=0.30$. There exist four phases: the Ising-SRO, $X Y$-SRO, $X Y$-SBO, and Ising-SBO. This situation is observed in the region 0 $<\gamma<\frac{1}{2}$, (see Sec. IV).

The Floquet analysis shows that depending on $\gamma$ the phase diagram spanned by $h$ and $\Omega$ is different. In the following section, using the mode expansion analysis, we will derive approximate phase diagrams for typical $\gamma$ regions giving three typical phase diagrams. 


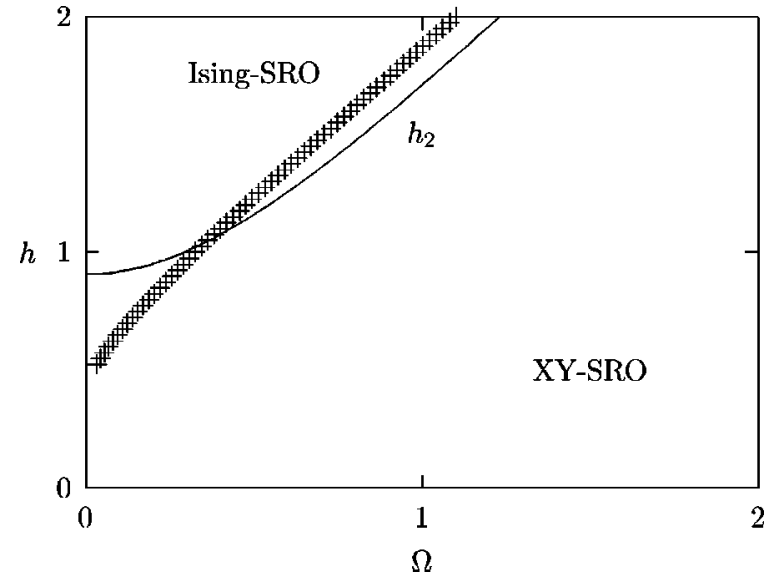

FIG. 6. Phase diagram for $\gamma=-0.05$. The transition curve indicated by $h_{2} \equiv h_{2}(\Omega)$ separates the Ising-SRO and the $X Y$-SRO phases. The meaning of symbols and solid line is the same as in Fig. 5.

\section{MODE EXPANSION ANALYSIS AND PHASE DIAGRAM}

First, we apply the Fourier expansion to $X(t)$ and $Y(t)$,

$$
X(t)=\sum_{n=-\infty}^{\infty} X_{n}(t) e^{i n \Omega t}, \quad Y(t)=\sum_{n=-\infty}^{\infty} Y_{n}(t) e^{i n \Omega t}
$$

where $X_{-n}=X_{n}^{*}$ and $Y_{-n}=Y_{n}^{*}$. We assume that the coefficients $\left\{X_{n}\right\}$ and $\left\{Y_{n}\right\}$ have weak time dependence whose time scales are much longer than $T$. Substituting Eq. (4.1) into Eqs. (2.6) and (2.7), and comparing the coefficients in both the right- and left-hand sides, we obtain

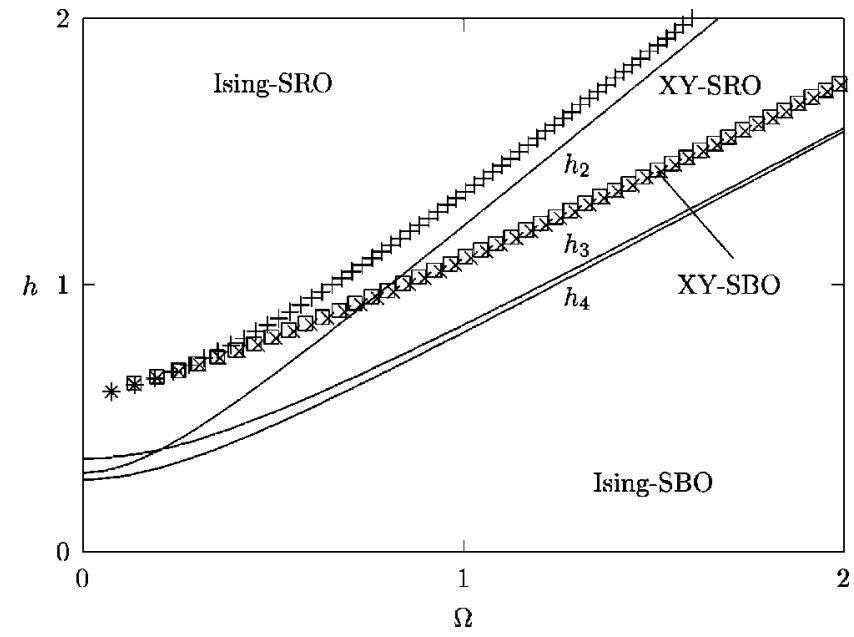

FIG. 7. Phase diagram for $\gamma=0.30$. There are three transition curves, $h_{2}, h_{3}$, and $h_{4}$, separating four phases. The $X Y$-SBO phase exists in a narrow region. The meaning of symbols and solid lines is the same as in Fig. 5.

$$
\begin{aligned}
\dot{X}_{n}+i n \Omega X_{n}= & (1+\gamma) X_{n}-\sum_{l} \sum_{m} X_{l} X_{m} X_{n-l-m} \\
& -\sum_{l} \sum_{m} Y_{l} Y_{m} X_{n-l-m} \\
& +\frac{h}{2}\left(\delta_{n, 1}+\delta_{n,-1}\right) \\
\dot{Y}_{n}+i n \Omega Y_{n}= & (1-\gamma) Y_{n}-\sum_{l} \sum_{m} Y_{l} Y_{m} Y_{n-l-m} \\
& -\sum_{l} \sum_{m} X_{l} X_{m} Y_{n-l-m} .
\end{aligned}
$$

In the Ising-type oscillation, the imaginary component $Y(t)$ vanishes, and therefore $Y_{l}=0(l=0, \pm 1, \pm 2, \ldots)$. In the SRO type oscillation, the relation (2.15) leads to $X_{l}=0$ ( $l$ $=0, \pm 2, \pm 4, \ldots)$.

As the simplest approximation, we take into account only the three modes $n=0$ and $n= \pm 1$. From Eqs. (4.2) and (4.3), the equations of motion for the mode $n=0$ are given by

$$
\begin{aligned}
\dot{X}_{0}= & \left(1+\gamma-X_{0}^{2}-6\left|X_{1}\right|^{2}-Y_{0}^{2}-2\left|Y_{1}\right|^{2}\right) X_{0} \\
& -2\left(X_{1} Y_{1}^{*}+X_{1}^{*} Y_{1}\right) Y_{0}, \\
\dot{Y}_{0}= & \left(1-\gamma-Y_{0}^{2}-6\left|Y_{1}\right|^{2}-X_{0}^{2}-2\left|X_{1}\right|^{2}\right) Y_{0} \\
& -2\left(Y_{1} X_{1}^{*}+Y_{1}^{*} X_{1}\right) X_{0} .
\end{aligned}
$$

Similarly, the equations for the mode $n=1$ are written as

$$
\begin{aligned}
\dot{X}_{1}+i \Omega X_{1}= & \left(1+\gamma-3 X_{0}^{2}-Y_{0}^{2}-3\left|X_{1}\right|^{2}-2\left|Y_{1}\right|^{2}\right) X_{1} \\
& -2 X_{0} Y_{0} Y_{1}-Y_{1}^{2} X_{1}^{*}+\frac{h}{2}, \\
\dot{Y}_{1}+i \Omega Y_{1}= & \left(1-\gamma-3 Y_{0}^{2}-X_{0}^{2}-3\left|Y_{1}\right|^{2}-2\left|X_{1}\right|^{2}\right) Y_{1} \\
& -2 X_{0} Y_{0} X_{1}-X_{1}^{2} Y_{1}^{*} .
\end{aligned}
$$

With the above simplest approximation, the stationary state $\left(\dot{X}_{j}=\dot{Y}_{j}=0\right)$ is determined by

$$
\begin{aligned}
0= & \left(1+\gamma-x_{0}^{2}-6\left|X_{1}^{s t}\right|^{2}-2\left|Y_{1}^{s t}\right|^{2}\right) x_{0} \\
& -2\left(X_{1}^{s t} Y_{1}^{s t} *+X_{1}^{s t} * Y_{1}^{s t}\right) y_{0}-x_{0} y_{0}^{2}, \\
0= & \left(1-\gamma-y_{0}^{2}-6\left|Y_{1}^{s t}\right|^{2}-2\left|X_{1}^{s t}\right|^{2}\right) y_{0} \\
& -2\left(X_{1}^{s t} Y_{1}^{s t} * X_{1}^{s t} * Y_{1}^{s t}\right) x_{0}-y_{0} x_{0}^{2},
\end{aligned}
$$

where $\left(X_{1}^{s t}, Y_{1}^{s t}\right)$ and $\left(x_{0}, y_{0}\right)$ represent the stationary solutions of $\left(X_{1}, Y_{1}\right)$ and $\left(X_{0}, Y_{0}\right)$, respectively.

The symmetry argument in Sec. II leads to the following results.

(i) If the fixed point $x_{0}=0$, and $y_{0}=0$ is stable, then the attractor is the Ising-SRO trajectory, and the relations $Y_{1}^{\text {st }}$ $=0$ and $\left(-i \Omega+1+\gamma-3\left|X_{1}^{s t}\right|^{2}\right) X_{1}^{s t}+h / 2=0$ hold. 
(ii) If the fixed points $x_{0} \neq 0$, and $y_{0}=0$ are stable, then the attractors are the Ising-SBO trajectories, and the relations $x_{0}= \pm \sqrt{1+\gamma-6\left|X_{1}^{s t}\right|^{2}}, \quad Y_{1}^{s t}=0 \quad$ and $\quad\left(-i \Omega+1+\gamma-3 x_{0}^{2}\right.$ $\left.-3\left|X_{1}^{s t}\right|^{2}\right) X_{1}^{s t}+h / 2=0$ hold.

(iii) If the fixed points $x_{0}=0$, and $y_{0} \neq 0$ are stable, then the attractors are the $X Y$-SRO trajectories, and the relations $y_{0}= \pm \sqrt{1-\gamma-2\left|X_{1}^{s t}\right|^{2}}, \quad Y_{1}^{s t}=0 \quad$ and $\quad\left(-i \Omega+1+\gamma-y_{0}^{2}\right.$ $\left.-3\left|X_{1}^{s t}\right|^{2}\right) X_{1}^{s t}+h / 2=0$ hold.

(iv) If the fixed points $x_{0} \neq 0$, and $y_{0} \neq 0$ are stable, the attractors are the $X Y$-SBO trajectories.

Now we consider the linear stability around the fixed points corresponding to the above stationary state. Noting that the mode $\left(X_{0}, Y_{0}\right)$ is relevant to the symmetry change of the oscillation, we assume that the relaxation time of the mode $\left(X_{1}, Y_{1}\right)$ is faster than that of $\left(X_{0}, Y_{0}\right)$, and we adiabatically eliminate $X_{1}$ and $Y_{1}$. With this approximation, $X_{1}$ and $Y_{1}$ are replaced by $X_{1}^{s t}$ and $Y_{1}^{s t}$, which are functions of $X_{0}$ and $Y_{0}$. Denoting the deviation from one of the fixed points as $\boldsymbol{x}=(\xi, \eta)$, i.e., the $X_{0}$ and $Y_{0}$ components being replaced with $X_{0}(t)=x_{0}+\xi(t)$ and $Y_{0}(t)=y_{0}+\eta(t)$ in Eqs. (4.4) and (4.5), we obtain the linearized equation $\dot{x}=\Lambda(t) \boldsymbol{x}$ for the deviation, where the matrix $\Lambda(t)$ has the elements.

$$
\begin{aligned}
& \Lambda_{11}=1+\gamma-3 x_{0}^{2}-y_{0}^{2}-6\left|X_{1}^{s t}\right|^{2}-2\left|Y_{1}^{s t}\right|^{2}, \\
& \Lambda_{12}=\Lambda_{21}=-2\left(x_{0} y_{0}+X_{1}^{s t} Y_{1}^{s t *}+X_{1}^{s t *} Y_{1}^{s t}\right), \\
& \Lambda_{22}=1-\gamma-3 y_{0}^{2}-x_{0}^{2}-6\left|Y_{1}^{s t}\right|^{2}-2\left|X_{1}^{s t}\right|^{2} .
\end{aligned}
$$

By using $\boldsymbol{x}(t)=e^{\lambda t} \boldsymbol{x}(0)[\boldsymbol{x}(0) \neq \mathbf{0}]$, the solutions of the characteristic equation for $\lambda$ are given by

$$
2 \lambda_{ \pm}=\Lambda_{11}+\Lambda_{22} \pm \sqrt{\left(\Lambda_{11}-\Lambda_{22}\right)^{2}+4 \Lambda_{12}^{2}} .
$$

It turns out that the characteristic equation always has a couple of real roots because of the symmetry of the matrix. On the transition curves, where the symmetry changes occur, the real part of the largest eigenvalue $\lambda_{+}$vanishes, and, simultaneously, $\Lambda_{12}$ vanishes from $x_{0} y_{0}=0$ and $Y_{1}^{s t}=0$. Therefore, on the transition curves we get the relations

$$
\begin{aligned}
& 1+\gamma-3 x_{0}^{2}-y_{0}^{2}-6\left|X_{1}^{s t}\right|^{2}=0 \text { for }-1+2 \gamma+4 y_{0}^{2}>0, \\
& 1-\gamma-3 y_{0}^{2}-x_{0}^{2}-2\left|X_{1}^{s t}\right|^{2}=0 \text { for }-1+2 \gamma+4 y_{0}^{2}<0 .
\end{aligned}
$$

The transition curves in the $(\Omega, h)$ space are determined by combining Eqs. (4.14) and (4.15) with the results in (i), (ii), and (iii). After some algebraic operations, we obtain the following results:

(i) For $\gamma \geqslant \frac{1}{2}$, the transition curve is given by

$$
h_{1}=\sqrt{\frac{2}{3}(1+\gamma)\left\{\Omega^{2}+\left(\frac{1+\gamma}{2}\right)^{2}\right\}} .
$$

For $h>h_{1}$, the Ising-SRO is stable, and the Ising-SBO is stable for $h<h_{1}$.

(ii) For $\gamma \leqslant 0$, the transition curve is written as

$$
h_{2}=\sqrt{2(1-\gamma)\left\{\Omega^{2}+\left(\frac{5 \gamma-1}{2}\right)^{2}\right\}} .
$$

For $h>h_{2}$, the Ising-SRO is stable, and the $X Y$-SRO is stable for $h<h_{2}$.

(iii) For $0<\gamma<\frac{1}{2}$, in addition to the same expression for $h_{2}$, the transition curves are represented by

$$
h_{3}=\sqrt{2 \gamma\left(\Omega^{2}+\frac{9}{4} \gamma^{2}\right)}, \quad h_{4}=\sqrt{2 \gamma\left\{\Omega^{2}+\left(\frac{11 \gamma-4}{2}\right)^{2}\right\}} .
$$

We find that the Ising-SRO, the $X Y$-SRO, the $X Y$-SBO, and the Ising-SBO are stable, respectively, for $h>h_{2}, h_{3}$ $<h<h_{2}, h_{4}<h<h_{3}$, and $h<h_{4}$. These results involve the fundamental behaviors expected from the symmetry argument. In Figs. 5, 6, and 7 the transition curves obtained above are compared with the results of the Floquet analysis. For the transition curve related to the Ising-type phases (Fig. 5), both results are quantitatively in agreement. However, for the transition curves associated with other types of DPTs, i.e., Figs. 6 and 7, the deviations between both results are rather large, although both results are qualitatively in agreement. This fact implies that the analysis based on the three modes approximation presented above is not accurate, particularly in a low-frequency region. The analysis should be carried out by taking into account higher-order modes.

\section{LANDAU EXPANSIONS NEAR THE DPT}

As is well known, in equilibrium thermodynamic phase transitions the Landau expansion is a powerful approach to clarify the universality class of the phase transition. In this section, we will carry out the Landau expansion with respect to the order parameter near DPTs to study the characteristics of the transitions.

\section{A. Near the DPT between Ising-SRO and Ising-SBO phases}

In this subsection, we consider the dynamics near the DPT between the Ising-SRO and the Ising-SBO, which is observed for $\gamma \geqslant \frac{1}{2}$. This transition can be discussed in a similar way to the DPT in the Ising spin system reported in Ref. [9].

Let $X_{R}(t)$ be the Ising-SRO trajectory, which is either stable or unstable. The Ising-SRO trajectory is numerically found as follows. If the Ising-SRO is stable, then for any initial condition one eventually obtains its trajectory. When the Ising-SRO is unstable, its trajectory can be found by constructing the stroboscopic map for the Ising-type equation of motion,

$$
\dot{X}(t)=\left(1+\gamma-X^{2}\right) X+h \cos (\Omega t) .
$$

For an arbitrary initial condition $X(0)$, the integration of the above equation until $t=T$ leads to a one-dimensional map $X(T)=g[X(0)]$, and, therefore,

$$
X\left(t_{n+1}\right)=g\left[X\left(t_{n}\right)\right] \quad\left(t_{n}=n T\right),
$$


where $g(X)$ is a nonlinear mapping function of $X$, whose form depends on both $h$ and $\Omega$. As was discussed in Ref. [9], if the Ising-SRO is stable, there exists one fixed point $X_{f}$ with the slope $\left|g^{\prime}\left(X_{f}\right)\right|$ less than unity. On the other hand, when the Ising-SRO is unstable and the Ising-SBOs are stable, there appear three fixed points, one of which, $X_{u}$ with $\left|g^{\prime}\left(X_{u}\right)\right|>1$, gives the unstable Ising-SRO, and others of which, $X_{s, 1}$ and $X_{s, 2}$ with $\left|g^{\prime}\left(X_{s, 1}\right)\right|=\left|g^{\prime}\left(X_{s, 2}\right)\right|<1$, correspond to the stable Ising-SBO. Numerical determination of $X_{u}$ gives the initial condition for the unstable Ising-SRO trajectory.

Using $X(t)=X_{R}(t)+x(t), Y(t)=0$, and

$$
x(t)=Q_{R}(t) \xi(t), \quad Q_{R}(t)=\exp \left[-3 \int_{0}^{t}\left\{X_{R}(s)^{2}-\overline{X_{R}^{2}}\right\} d s\right],
$$

we get

$$
\dot{\xi}(t)=\lambda \xi-3 X_{R}(t) Q_{R}(t) \xi^{2}-Q_{R}(t)^{2} \xi^{3},
$$

where

$$
\lambda=1+\gamma-3 \overline{X_{R}^{2}}
$$

is the Floquet exponent of the Ising-SRO. When the IsingSRO is stable (unstable), $\lambda$ is negative (positive). The $\bar{A}$ stands for the time average of $A(t)$ over one period $T$.

Near the DPT, $\lambda \approx 0$, and therefore the time scale $|\lambda|^{-1}$ of $\xi$ is quite long. We can replace the coefficient of $\xi$ in Eq. (5.4) by its time average over one period $T$ of the external field. One thus obtains

$$
\dot{\xi}(t)=\lambda \xi-c \xi^{3} \quad\left(c=\overline{Q_{R}^{2}}\right) .
$$

Here we used the fact that the symmetry $X_{R}(t)=-X_{R}(t$ $+T / 2$ ) leads to $\overline{X_{R} Q_{R}}=0$. One thus finds that $\xi_{s}$, the average of $\xi$ over one period $T$, is of order $\sqrt{\lambda} \propto \sqrt{\Omega-\Omega_{1}}$ for $\Omega$ slightly beyond $\Omega_{1}$, the DPT point between the Ising-SRO and the Ising-SBO. The comparison of the trajectory obtained from Eq. (5.3) and Eq. (5.6) with that exactly obtained from Eq. (2.5) is made in Fig. 8. From the symmetry there exist two Ising-SBO trajectories for $\bar{X}>0$ and $\bar{X}<0$. In the figure, one of them corresponding to that for $\bar{X}>0$ is drawn. The period of $x$ derived from Eqs. (5.3) and (5.6) is $T / 2$, and the trajectory is surrounded by that obtained by numerically integrating Eq. (2.5). As the transition point is approached, the two trajectories tend to coincide with each other.

One should note that when the spatial variation of the order parameter exists, Eq. (5.6) should be replaced by

$$
\dot{\xi}(\boldsymbol{r}, t)=\lambda \xi-c \xi^{3}+\nabla^{2} \xi
$$

Therefore, the DPT between the Ising-SRO and Ising-SBO and the Ising-SBO belongs to the same universality class as that of the Ising spin system in thermal equilibrium [9]. As will be discussed in Sec. VI Eq. (5.7) has the Néel wall solution.

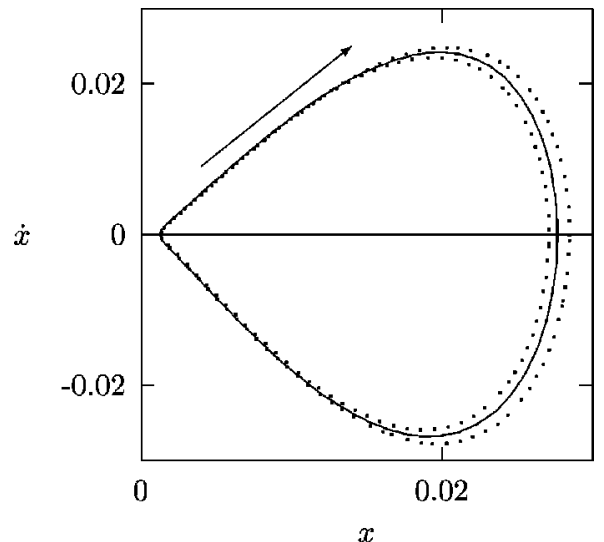

FIG. 8. Comparison of the trajectory obtained with the Landau expansion (solid line) from Eq. (5.6) with the exact one (broken line) from Eq. (2.5) near the DPT between Ising-SRO and IsingSBO for the parameter values, $\gamma=0.55, h=1.0$, and $\Omega=0.52557$ $=\Omega_{1}+10^{-4}$, and $x(t)=X(t)-X_{R}(t), Y(t)=0$. The arrow shows the movement of the phase point in the course of time.

\section{B. Near the DPT between Ising-type and $X Y$-type oscillations}

In this subsection, we consider the critical dynamics near the DPT between the Ising-type motion and the $X Y$-type motion observed for $\gamma<\frac{1}{2}$, i.e., (i) the DPT between the Ising-SRO and the $X Y$-SRO, and (ii) the DPT between the Ising-SBO and the $X Y$-SBO.

Let $X_{I}(t)$ be the motion with the Ising-type trajectory, either SRO and SBO. Near their DPTs, the insertion of $X(t)=X_{I}(t)+x(t), Y(t)=y(t)$ with

$$
\begin{aligned}
& x(t)=Q_{3}(t) \xi(t), \quad y(t)=Q_{1}(t) \eta(t), \\
& Q_{n}(t) \equiv \exp \left[-n \int_{0}^{t}\left\{X_{I}(s)^{2}-\overline{X_{I}^{2}}\right\} d s\right]
\end{aligned}
$$

into Eq. (2.5) yields

$$
\begin{aligned}
\xi(t)= & \lambda_{1} \xi-3 X_{I}(t) Q_{3}(t) \xi^{2}-X_{I}(t) Q_{-1}(t) \eta^{2} \\
- & {\left[Q_{6}(t) \xi^{2}+Q_{2}(t) \eta^{2}\right] \xi } \\
\dot{\eta}(t)= & \lambda_{2} \eta-2 X_{I}(t) Q_{3}(t) \xi \eta \\
& -\left[Q_{6}(t) \xi^{2}+Q_{2}(t) \eta^{2}\right] \eta
\end{aligned}
$$

where

$$
\lambda_{1}=1+\gamma-3 \overline{X_{I}^{2}}, \quad \lambda_{2}=1-\gamma-\overline{X_{I}^{2}} .
$$

One should note that near the DPT, $\lambda_{2} \approx 0$ and $\lambda_{1}$ keeps negative, and therefore $\xi$ decays faster than $\eta$. By taking into account this fact and using the estimation $\xi=O\left(\left|\lambda_{2}\right|\right)$ and $\eta=O\left(\sqrt{\left|\lambda_{2}\right|}\right)$, Eqs. (5.10) and (5.11) are simplified into

$$
\begin{gathered}
\dot{\xi}(t)=\lambda_{1} \xi-X_{I}(t) Q_{-1}(t) \eta^{2}, \\
\dot{\eta}(t)=\lambda_{2} \eta-2 X_{I}(t) Q_{3}(t) \xi \eta-Q_{2}(t) \eta^{3} .
\end{gathered}
$$



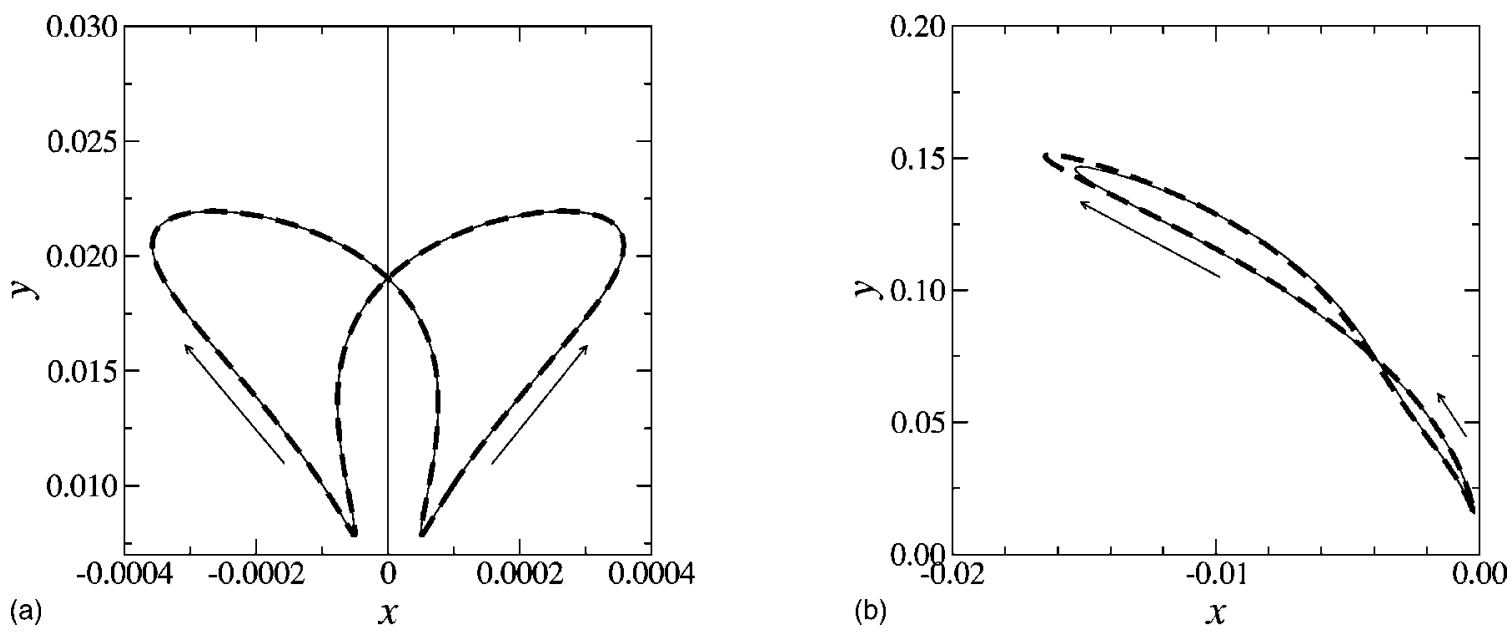

FIG. 9. Comparison of the trajectories obtained with the Landau expansion (solid lines) Eqs. (5.13) and (5.14) with the exact ones (broken lines) from Eq. (2.5) near the DPT between the Ising-type oscillation and the $X Y$-type oscillation for the parameter values (a) $\gamma$ $=0.30, h=1.0$, and $\Omega=0.65630=\Omega_{4}+10^{-4}$; and (b) $\gamma=0.30, h=1.0$, and $\Omega=0.85903=\Omega_{4}-10^{-4}$, and $x(t)=X(t)-X_{I}(t), y(t)$ $=Y(t)-Y_{I}(t)$. Arrows show the movement of the phase points in the course of time.

Near the DPT point between the Ising-SRO and the $X Y$-SRO, the Ising-SRO trajectory is obtained as follows. When the Ising-SRO is stable, one immediately gets its trajectory by simply integrating the original equation of motion for an arbitrary initial condition.On the contrary, when it is unstable in the region where the $X Y$-SRO stably exists, we can obtain the unstable Ising-SRO trajectory by integrating the equation of motion starting from an initial phase point on the axis $Y=0$. The comparison of the trajectory obtained from Eqs. (5.8), (5.9), (5.13), and (5.14) with that from Eq. (2.5) for $\gamma>0$ is made in Fig. 9(a). The figure shows the difference of the trajectory from the unstable orbit $\left(X_{I}, 0\right)$ just after the DPT between the Ising-SRO and the $X Y$-SRO. In the figure, the trajectory for $\bar{Y}>0$ of two equivalent $X Y$-SRO attractors is shown.

When the spatial variation is taken into account, we should add the $\nabla^{2} \xi$ and $\nabla^{2} \eta$ terms, respectively, in Eqs. (5.13) and (5.14). If we use the approximation to replace the coefficients $X_{I}(t) Q_{n}(t)(n=-1,3)$ and $Q_{2}(t)$, respectively, by their time averages 0 and $c_{2}(>0)$, we get coupled equations of motion for $\xi(\boldsymbol{r}, t)$ and $\eta(\boldsymbol{r}, t)$. Since $\lambda_{1}<0$ near the transition point, $\lambda_{2} \approx 0$, and $\xi$ eventually vanishes. Thus one arrives at

$$
\dot{\eta}(\boldsymbol{r}, t)=\lambda_{2} \eta-c_{2} \eta^{3}+\nabla^{2} \eta .
$$

This also confirms that the present transition also belongs to the universality class of the Ising spin system in thermal equilibrium.

Figure 9 (b) shows the difference of the trajectory from the unstable orbit $\left(X_{I}, 0\right)$ just after the DPT between the Ising$\mathrm{SBO}$ and the $X Y$-SBO, where the Ising-SBO trajectory is obtained by integrating the equation of motion starting from a phase point on $Y=0$. In the figure, the trajectory for $\bar{X}$ $>0$ and $\bar{Y}>0$ of four equivalent $X Y$-SBO attractors is shown. We find that the above approximations explain the exact dynamics quite well near the DPTs. It should be noted that the difference between the exact trajectory and the approximate one is more remarkable near the Ising-SBO vs $X Y$-SBO transition than near the Ising-SRO vs $X Y$-SRO transition. This is because in the former the Ising-SBO vs $X Y$-SBO transition is close to the $X Y$-SRO vs $X Y$-SBO transition, and the transition property of the $X Y$-SRO vs $X Y$-SBO affects the Ising-SBO vs $X Y$-SBO transition. In the above simple treatment, however, we did not take into account the inter-relation of each other.

In the case when there exists a spatial variation of the order parameter, we can repeat the previous treatment for the DPT between the Ising-SRO and the $X Y$-SRO. By using $a_{n}$ $\equiv \overline{X_{I} Q_{n}}$, the resulting equations obtained in this way are

$$
\begin{gathered}
\dot{\xi}(\boldsymbol{r}, t)=\lambda_{1} \xi-a_{-1} \eta^{2}+\nabla^{2} \xi, \\
\dot{\eta}(\boldsymbol{r}, t)=\lambda_{2} \eta-2 a_{3} \xi \eta-c_{2} \eta^{3}+\nabla^{2} \eta .
\end{gathered}
$$

One should note that in the present case $a_{n}$ does not vanish because the reference trajectory does not satisfy $X_{I}(t)=$ $-X_{I}(t+T / 2)$. Since $\lambda_{1}<0$, one may adiabatically eliminate $\xi$, the insertion of which into Eq. (5.17) leads to

$$
\dot{\eta}(\boldsymbol{r}, t)=\lambda_{2} \eta-\left(c_{2}+\frac{2 a_{-1} a_{3}}{\lambda_{1}}\right) \eta^{3}+\nabla^{2} \eta .
$$

This equation has the meanings when the inequality $c_{2}$ $+2 a_{-1} a_{3} / \lambda_{1}>0$ holds. It is worth noting that although Eqs. (5.16) and (5.17) are different from the type of GinzburgLandau equation (5.15), the enhancement of the difference of time scales sufficiently near DPT confirms that the reduced equations (5.16) and (5.17) again can be reduced to the Ginzburg-Landau equation (5.18). This implies that the present transition again belongs to the universality class of the Ising spin system. 


\section{SPATIAL VARIATION AND DOMAIN WALLS}

There are two stable oscillating states both in the IsingSBO phase and in the $X Y$-SRO phase, and four stable oscillating states in the $X Y$-SBO phase. In this section, deriving the fundamental equations of motion describing the spatial variation of a spin variable (order parameter), we will discuss domain walls connecting a couple of the stable oscillating states in one dimension.

\section{A. Domain walls in the Ising-SBO phase}

Let $C_{I B}^{ \pm}$denote spatially uniform period- $T$ limit cycles in the Ising-SBO phase, where $C_{I B}^{+}$and $C_{I B}^{-}$indicate the oscillations corresponding to $\bar{X}>0$ and $\bar{X}<0$, respectively. Let $X_{ \pm}(t)=\left[X_{ \pm}(t), 0\right]$ stand for the spin variable of the IsingSBO trajectory $C_{I B}^{ \pm}$at time $t$. We introduce the quantity $[a(t), b(t)]$ which is identical to $(+1,0)[(-1,0)]$ when the phase point is on $C_{I B}^{+}\left(C_{I B}^{-}\right)$at time $t$. This quantity can be extended to the case when there exists spatial variation of spin variables. Namely, the variables $a(\boldsymbol{r}, t)$ and $b(\boldsymbol{r}, t)$ defined by

$$
\begin{gathered}
X(\boldsymbol{r}, t)=\frac{1+a(\boldsymbol{r}, t)}{2} X_{+}(t)+\frac{1-a(\boldsymbol{r}, t)}{2} X_{-}(t), \\
Y(\boldsymbol{r}, t)=\frac{X_{+}(t)-X_{-}(t)}{2} b(\boldsymbol{r}, t)
\end{gathered}
$$

measure how close the local order parameter $[X(\boldsymbol{r}, t), Y(\boldsymbol{r}, t)]$ is to the trajectory of either $C_{I B}^{+}$or $C_{I B}^{-}$. The insertion of Eqs. (6.1) and (6.2) into Eq. (2.1) yields

$$
\begin{gathered}
\dot{a}(\boldsymbol{r}, t)=3 f(t)+g(t) a-f(t)\left(3 a^{2}+b^{2}\right)-g(t)\left(a^{2}+b^{2}\right) a \\
+\nabla^{2} a, \\
\dot{b}(\boldsymbol{r}, t)=h(t) b-2 f(t) a b-g(t)\left(a^{2}+b^{2}\right) b+\nabla^{2} b,
\end{gathered}
$$

with

$$
\begin{gathered}
f(t)=\frac{1}{4}\left[X_{+}(t)^{2}-X_{-}(t)^{2}\right], \\
g(t)=\frac{1}{4}\left[X_{+}(t)-X_{-}(t)\right]^{2}, \\
h(t)=\frac{1}{4}\left[3 X_{+}(t)^{2}+2 X_{+}(t) X_{-}(t)+3 X_{-}(t)^{2}-8 \gamma\right] .
\end{gathered}
$$

In order to see the long-time behavior of $a$ and $b$, we use the approximation to replace temporally periodic coefficients such as $f(t)$ and $g(t)$ in the above equations of motion by their time averages over one period. The resulting equations are given as

$$
\dot{a}(\boldsymbol{r}, t)=\bar{g} a-\bar{g}\left(a^{2}+b^{2}\right) a+\nabla^{2} a,
$$

$$
\dot{b}(\boldsymbol{r}, t)=\bar{h} b-\bar{g}\left(a^{2}+b^{2}\right) b+\nabla^{2} b .
$$

Here we used the fact that the symmetry $X_{-}(t)=-X_{+}(t$ $+T / 2$ ) yields $\bar{f}=0$. In addition to the spatially uniform solutions $a= \pm 1, b=0$, one finds that Eqs. (6.6) and (6.7) possess stationary domain wall solutions in a one-dimensional system with the boundary condition $[a(+\infty), a(-\infty)]=$ $(+1,-1)$ or $(-1,+1)$ and $b( \pm \infty)=0$. We find that there are two types of domain walls. The first is the Néel wall, $z$ being the spatial coordinate,

$$
a(z)= \pm \tanh \left(\frac{z-z_{0}}{\xi}\right), \quad b(z)=0, \quad \xi=\sqrt{\frac{2}{g}}
$$

where $z_{0}$ is the position of the wall. One should note that $\bar{g}$ is always positive. The second is the Bloch wall

$$
\begin{gathered}
a(z)= \pm \tanh \left(\frac{z-z_{0}}{\xi}\right), \quad b(z)= \pm p \operatorname{sech}\left(\frac{z-z_{0}}{\xi}\right), \\
\xi=\sqrt{\frac{1}{\bar{g}-\bar{h}}}, \quad p=\sqrt{\frac{2 \bar{h}-\bar{g}}{\bar{g}}} .
\end{gathered}
$$

Of course, this solution has the meaning only when the inequality $\bar{h}<\bar{g}<2 \bar{h}$ holds. We confirmed that for the parameter values we used, except the DPT, this inequality holds. However, as the parameter value is approached to the transition curve $h_{1}$ (Fig. 5), $\bar{g}<\bar{h}$ is satisfied. This fact implies that near the DPT only the Néel wall exists.

It turns out that these domain wall solutions reduce to the solutions in the absence of external fields in the zero field limit $h \rightarrow 0, X_{ \pm}(t) \rightarrow \pm \sqrt{1+\gamma}$ [Eq. (2.8)], i.e., we find $f(t)$ $\rightarrow 0, g(t) \rightarrow 1+\gamma$, and $h(t) \rightarrow 1-\gamma$ in that limit, and thus the solutions (6.8) and (6.9) agree with the walls in the zero field.

\section{B. Domain walls in the $X Y$-SRO phase}

Let $C_{X Y R}{ }^{ \pm}$denote spatially uniform period- $T$ limit cycles in the $X Y$-SRO phase, where $C_{X Y R}{ }^{+}$and $C_{X Y R}{ }^{-}$indicate the oscillations corresponding to $\bar{Y}>0$ and $\bar{Y}<0$, respectively. Let $\boldsymbol{X}_{ \pm}(t)=\left[X_{0}(t), Y_{ \pm}(t)\right]$ denote the spin variable of the $X Y$-SRO trajectory $C_{X Y R}{ }^{ \pm}$at time $t$. We introduce the quantity $[a(t), b(t)]$ which takes the value $(0,+1)[(0,-1)]$ when the phase point is on $C_{X Y R}{ }^{+}\left(C_{X Y R}{ }^{-}\right)$at time $t$. This quantity can be also extended to the case when spatial variation of spin variables is present. Namely, the variables $a(\boldsymbol{r}, t)$ and $b(\boldsymbol{r}, t)$ defined by

$$
\begin{aligned}
X(\boldsymbol{r}, t) & =X_{0}(t)+\frac{Y_{+}(t)-Y_{-}(t)}{2} a(\boldsymbol{r}, t) \\
& =X_{0}(t)+Y_{+}(t) a(\boldsymbol{r}, t),
\end{aligned}
$$




$$
\begin{aligned}
Y(\boldsymbol{r}, t) & =\frac{1+b(\boldsymbol{r}, t)}{2} Y_{+}(t)+\frac{1-b(\boldsymbol{r}, t)}{2} Y_{-}(t) \\
& =Y_{+}(t) b(\boldsymbol{r}, t)
\end{aligned}
$$

measure how close the local order parameter $[X(\boldsymbol{r}, t), Y(\boldsymbol{r}, t)]$ is to either $C_{X Y R}{ }^{+}$or $C_{X Y R}{ }^{-}$. Here we used the fact that $Y_{-}(t)=-Y_{+}(t)$. Inserting Eqs. (6.11) and (6.12) into Eq. (2.1), we obtain

$$
\begin{gathered}
\dot{a}(\boldsymbol{r}, t)=h(t)+[g(t)-f(t)] a-h(t)\left(3 a^{2}+b^{2}\right) \\
-g(t)\left(a^{2}+b^{2}\right) a+\nabla^{2} a, \\
\dot{b}(\boldsymbol{r}, t)=g(t) b-2 h(t) a b \\
-g(t)\left(a^{2}+b^{2}\right) b+\nabla^{2} b,
\end{gathered}
$$

with

$$
\begin{gathered}
f(t)=2\left[X_{0}(t)^{2}-\gamma\right], \\
g(t)=Y_{+}(t)^{2}, \\
h(t)=X_{0}(t) Y_{+}(t) .
\end{gathered}
$$

In order to see the long-time behavior of $a$ and $b$, we use the approximation to replace temporally periodic coefficients such as $f(t)$ and $g(t)$ by their time average values over one period. The resulting equations are written as

$$
\begin{gathered}
\dot{a}(\boldsymbol{r}, t)=(\bar{g}-\bar{f}) a-\bar{g}\left(a^{2}+b^{2}\right) a+\nabla^{2} a, \\
\dot{b}(\boldsymbol{r}, t)=\bar{g} b-\bar{g}\left(a^{2}+b^{2}\right) b+\nabla^{2} b .
\end{gathered}
$$

Here we used the fact that the symmetries $X_{0}(t)=-X_{0}(t$ $+T / 2)$ and $Y_{+}(t)=Y_{+}(t+T / 2)$ lead to $\bar{h}=0$.

Consider a one-dimensional system with the coordinate $z$. It is easy to see that in addition to the stable spatially uniform solutions $a=0,|b|=1$, Eqs. (6.16) and (6.17) possess stationary domain wall solutions for the boundary condition $a( \pm \infty)=0$ and $[b(+\infty), b(-\infty)]=(+1,-1)$ or $(-1,+1)$. Particularly, we find two types of domain walls. The first is the Néel wall

$$
a(z)=0, \quad b(z)= \pm \tanh \left(\frac{z-z_{0}}{\xi}\right), \quad \xi=\sqrt{\frac{2}{\bar{g}}} .
$$

The second is the Bloch wall

$$
\begin{gathered}
a(z)= \pm p \operatorname{sech}\left(\frac{z-z_{0}}{\xi}\right), \quad b(z)= \pm \tanh \left(\frac{z-z_{0}}{\xi}\right) \\
\xi=\sqrt{\frac{1}{\bar{f}},} \quad p=\sqrt{\frac{\bar{g}-2 \bar{f}}{\bar{g}}}
\end{gathered}
$$

Needless to say, $\bar{g}>0$. For the parameter values we used, we find $\bar{f}>0$ and $\bar{g}>2 \bar{f}$. Near the DPT, we find $\bar{g}<2 \bar{f}$, which implies that only the Néel wall is observed near the DPT.

The above domain walls are reduced to those without an oscillating external field. This can be shown as follows. Since in the limit $h \rightarrow 0, X_{0}(t) \rightarrow 0, Y_{ \pm}(t) \rightarrow \pm \sqrt{1-\gamma}$ [Eq. (2.10)], we find $f(t) \rightarrow-2 \gamma, g(t) \rightarrow 1-\gamma$, and $h(t) \rightarrow 0$ in the zero field limit. Inserting the Néel wall solution (6.18) into Eqs. (6.11) and (6.12), we obtain the Néel wall in the zero field. In a similar way, inserting the Bloch wall solution (6.19) into Eqs. (6.11) and (6.12), one arrives at the Bloch wall solution without an oscillating external field.

\section{Domain walls across the $X$ axis in the $X Y$-SBO phase}

Let $C_{X Y B j}(j=1,2,3,4)$ be spatially uniform period- $T$ limit cycles in the $X Y$-SBO phase. Here $C_{X Y B 1}$ denotes the oscillation for $\bar{X}>0$ and $\bar{Y}>0, C_{X Y B 2}$ for $\bar{X}>0$ and $\bar{Y}<0$, $C_{X Y B 3}$ for $\bar{X}<0$ and $\bar{Y}<0$, and $C_{X Y B 4}$ for $\bar{X}<0$ and $\bar{Y}>0$. The quantity $\boldsymbol{X}_{j}(t)=\left[X_{j}(t), Y_{j}(t)\right]$ stands for the spin variable of the $X Y$-SBO trajectory $C_{X Y B j}$. Since the local oscillation is one of four limit cycles $C_{X Y B j}(j=1,2,3,4)$, we have to take into account the coexistence of these four oscillating states. However, for simplicity, in this paper we discuss only the coexistence of two of the four oscillatory states.

First we consider the domain walls connecting $C_{X Y B 1}$ and $C_{X Y B 2}$. From the symmetry of the solutions, those for $C_{X Y B 4}$ and $C_{X Y B 3}$ can be considered in the same way as those for $C_{X Y B 1}$ and $C_{X Y B 2}$. Let $[a(t), b(t)]$ be the quantity that takes the value $(0,+1)[(0,-1)]$ when the phase point is on $C_{X Y B 1}\left(C_{X Y B 2}\right)$ at time $t . a(t)$ and $b(t)$ are extended so as to include the position dependence when there exists spatial variation. Namely, the quantities $a(\boldsymbol{r}, t)$ and $b(\boldsymbol{r}, t)$ defined by

$$
\begin{gathered}
X(\boldsymbol{r}, t)=X_{1}(t)+\frac{Y_{1}(t)-Y_{2}(t)}{2} a(\boldsymbol{r}, t)=X_{1}(t)+Y_{1}(t) a(\boldsymbol{r}, t), \\
Y(\boldsymbol{r}, t)=\frac{1+b(\boldsymbol{r}, t)}{2} Y_{1}(t)+\frac{1-b(\boldsymbol{r}, t)}{2} Y_{2}(t)=Y_{1}(t) b(\boldsymbol{r}, t)
\end{gathered}
$$

measure how close the local order parameter $[X(\boldsymbol{r}, t), Y(\boldsymbol{r}, t)]$ is to either $C_{X Y B 1}$ or $C_{X Y B 2}$. Here we used the fact that $Y_{2}(t)=-Y_{1}(t)$. Inserting Eqs. (6.21) and (6.22) into Eq. (2.1), after some algebra we get

$$
\begin{gathered}
\dot{a}(\boldsymbol{r}, t)=h(t)+[g(t)-f(t)] a-h(t)\left(3 a^{2}+b^{2}\right) \\
-g(t)\left(a^{2}+b^{2}\right) a+\nabla^{2} a, \\
\dot{b}(\boldsymbol{r}, t)=g(t) b-2 h(t) a b \\
-g(t)\left(a^{2}+b^{2}\right) b+\nabla^{2} b,
\end{gathered}
$$

with

$$
f(t)=2\left[X_{1}(t)^{2}-\gamma\right],
$$




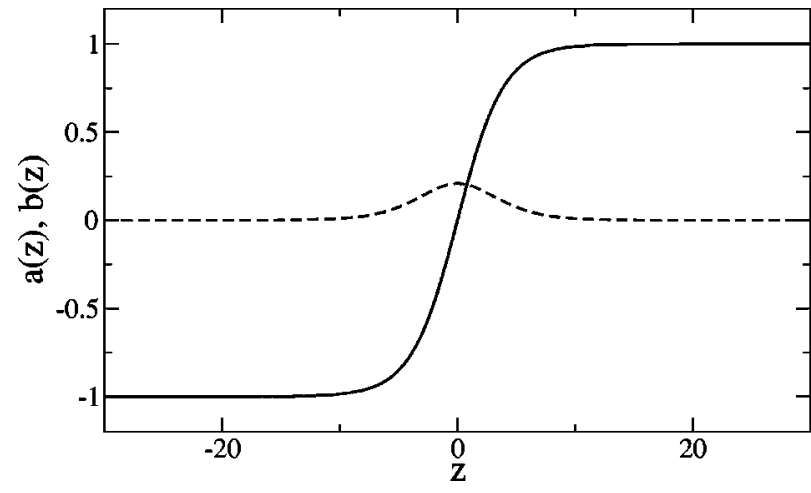

FIG. 10. Numerical solution of Eqs. (6.26) and (6.27), which corresponds to the boundary condition $a( \pm \infty)=0, b( \pm \infty)= \pm 1$. Solid and broken lines, respectively, denote $b(z)$ and $a(z)$. The parameters are $\gamma=0.30, h=1.0, \Omega=0.85$

$$
\begin{gathered}
g(t)=Y_{1}(t)^{2}, \\
h(t)=X_{1}(t) Y_{1}(t) .
\end{gathered}
$$

In order to observe the long-time behavior of $a$ and $b$, we replace temporally periodic coefficients such as $f(t)$ and $g(t)$ by their time averages. The resulting equations are given as

$$
\begin{gathered}
\dot{a}(\boldsymbol{r}, t)=\bar{h}+(\bar{g}-\bar{f}) a-\bar{h}\left(3 a^{2}+b^{2}\right)-\bar{g}\left(a^{2}+b^{2}\right) a+\nabla^{2} a, \\
\dot{b}(\boldsymbol{r}, t)=\bar{g} b-2 \bar{h} a b-\bar{g}\left(a^{2}+b^{2}\right) b+\nabla^{2} b .
\end{gathered}
$$

Consider a one-dimensional system with the spatial coordinate $z$. Figure 10 shows the steady-state pattern obtained from numerical integration of the above equations of motion with the boundary conditions $|a( \pm \infty)|=0,|b( \pm \infty)|=1$ for the parameters $\gamma=0.30, h=1.0, \Omega=0.85$. We conclude that the solution observed in this case is thus the Bloch type wall.

\section{Domain wall across the $Y$ axis in the $X Y$-SBO phase}

Next let us consider the coexistence of $C_{X Y B 1}$ and $C_{X Y B 4}$. From the symmetry of the solutions, domain walls connecting $C_{X Y B 2}$ and $C_{X Y B 3}$ can be studied in the same way as those in the preceding subsection. Let $[a(t), b(t)]$ be $(+1$, $+1)[(-1,-1)]$ when the phase point is on $C_{X Y B 1}\left(C_{X Y B 4}\right)$ at time $t$. The quantities $a(t)$ and $b(t)$ are extended to the case when there exists spatial variation. The quantities $a(\boldsymbol{r}, t)$ and $b(\boldsymbol{r}, t)$ defined by

$$
\begin{aligned}
& X(\boldsymbol{r}, t)=\frac{1+a(\boldsymbol{r}, t)}{2} X_{1}(t)+\frac{1-a(\boldsymbol{r}, t)}{2} X_{4}(t), \\
& Y(\boldsymbol{r}, t)=\frac{1+b(\boldsymbol{r}, t)}{2} Y_{1}(t)+\frac{1-b(\boldsymbol{r}, t)}{2} Y_{4}(t)
\end{aligned}
$$

measure how close the local order parameter $[X(\boldsymbol{r}, t), Y(\boldsymbol{r}, t)]$ is to either $C_{X Y B 1}$ or $C_{X Y B 4}$.
Inserting Eqs. (6.28) and (6.29) into Eq. (2.1), after some algebra we get

$$
\begin{aligned}
\dot{a}(\boldsymbol{r}, t)= & f_{1}(t)+f_{2}(t) a-f_{3}(t) b-3 f_{4}(t) a^{2}-f_{5}(t) b^{2}(t) \\
& -2 f_{6}(t) a b-f_{7}(t) a^{3}-f_{8}(t) a b^{2}+\nabla^{2} a, \\
\dot{b}(\boldsymbol{r}, t)= & g_{1}(t)+g_{2}(t) b-g_{3}(t) a-3 f_{6}(t) b^{2}-g_{5}(t) a^{2}(t) \\
& -2 f_{4}(t) b a-f_{8}(t) b^{3}-f_{7}(t) b a^{2}+\nabla^{2} b
\end{aligned}
$$

with

$$
\begin{aligned}
f_{1}(t)= & \frac{3}{4}\left[X_{1}(t)^{2}-X_{4}(t)^{2}\right] \\
& -\frac{\left[X_{1}(t)+X_{4}(t)\right]\left[Y_{1}(t)+Y_{4}(t)\right]^{2}}{4\left[X_{1}(t)-X_{4}(t)\right]} \\
& +\frac{Y_{1}(t)^{2} X_{1}(t)+Y_{4}(t)^{2} X_{4}(t)}{X_{1}(t)-X_{4}(t)}
\end{aligned}
$$

$$
\begin{aligned}
f_{2}(t)= & \frac{1}{4}\left[X_{1}(t)-X_{4}(t)\right]^{2}-\frac{1}{4}\left[Y_{1}(t)+Y_{4}(t)\right]^{2} \\
& +\frac{Y_{1}(t)^{2} X_{1}(t)-Y_{4}(t)^{2} X_{4}(t)}{X_{1}(t)-X_{4}(t)}
\end{aligned}
$$$$
f_{3}(t)=\frac{\left[X_{1}(t)+X_{4}(t)\right]\left[Y_{1}(t)^{2}-Y_{4}(t)^{2}\right]}{2\left[X_{1}(t)-X_{4}(t)\right]},
$$

$$
f_{4}(t)=\frac{1}{4}\left[X_{1}(t)^{2}-X_{4}(t)^{2}\right]
$$$$
f_{5}(t)=\frac{\left[X_{1}(t)+X_{4}(t)\right]\left[Y_{1}(t)-Y_{4}(t)\right]^{2}}{4\left[X_{1}(t)-X_{4}(t)\right]},
$$$$
f_{6}(t)=\frac{1}{4}\left[Y_{1}(t)^{2}-Y_{4}(t)^{2}\right],
$$

$$
\begin{aligned}
& f_{7}(t)=\frac{1}{4}\left[X_{1}(t)-X_{4}(t)\right]^{2}, \quad f_{8}(t)=\frac{1}{4}\left[Y_{1}(t)-Y_{4}(t)\right]^{2}, \\
& g_{1}(t)= \frac{3}{4}\left[Y_{1}(t)^{2}-Y_{4}(t)^{2}\right] \\
&-\frac{\left[Y_{1}(t)+Y_{4}(t)\right]\left[X_{1}(t)+X_{4}(t)\right]^{2}}{4\left[Y_{1}(t)-Y_{4}(t)\right]} \\
&+\frac{X_{1}(t)^{2} Y_{1}(t)+X_{4}(t)^{2} Y_{4}(t)}{Y_{1}(t)-Y_{4}(t)}, \\
& g_{2}(t)=\frac{1}{4}\left[Y_{1}(t)-Y_{4}(t)\right]^{2}-\frac{1}{4}\left[X_{1}(t)+X_{4}(t)\right]^{2} \\
&+ \\
&+\frac{X_{1}(t)^{2} Y_{1}(t)-X_{4}(t)^{2} Y_{4}(t)}{Y_{1}(t)-Y_{4}(t)},
\end{aligned}
$$




$$
\begin{gathered}
g_{3}(t)=\frac{\left[Y_{1}(t)+Y_{4}(t)\right]\left[X_{1}(t)^{2}-X_{4}(t)^{2}\right]}{2\left[Y_{1}(t)-Y_{4}(t)\right]}, \\
g_{5}(t)=\frac{\left[Y_{1}(t)+Y_{4}(t)\right]\left[X_{1}(t)-X_{4}(t)\right]^{2}}{4\left[Y_{1}(t)-Y_{4}(t)\right]} .
\end{gathered}
$$

Here the coefficients satisfy

$$
\begin{aligned}
f_{2}(t)-f_{3}(t) & =g_{2}(t)-g_{3}(t) \\
& =f_{7}(t)+f_{8}(t) \\
& =\frac{1}{4}\left[X_{1}(t)-X_{4}(t)\right]^{2}+\frac{1}{4}\left[Y_{1}(t)-Y_{4}(t)\right]^{2} \\
& \equiv h(t) .
\end{aligned}
$$

In order to see the long-time behavior of $a$ and $b$, we replace temporally periodic coefficients $\left\{f_{i}(t)\right\}$ and $\left\{g_{i}(t)\right\}$ of the above equations of motion by their time averages. The resulting equations are found to be

$$
\begin{aligned}
& \dot{a}(\boldsymbol{r}, t)=\bar{f}_{2}(a-b)+\left(\bar{f}_{7}+\bar{f}_{8}\right) b-\left(\bar{f}_{7} a^{2}+\bar{f}_{8} b^{2}\right) a+\nabla^{2} a, \\
& \dot{b}(\boldsymbol{r}, t)=\bar{g}_{2}(b-a)+\left(\bar{f}_{7}+\bar{f}_{8}\right) a-\left(\bar{f}_{7} a^{2}+\bar{f}_{8} b^{2}\right) b+\nabla^{2} b .
\end{aligned}
$$

Here we used the fact that the symmetry $\left[X_{4}(t), Y_{4}(t)\right]=$ $\left[-X_{1}(t+T / 2), Y_{1}(t+T / 2)\right]$ leads to $\bar{f}_{1}=\bar{f}_{4}=\bar{f}_{5}=\bar{f}_{6}=\bar{g}_{1}$ $=\bar{g}_{5}=0$. We carried out numerical integration of Eqs. (6.34) and (6.35) for $\gamma=0.3$ and $h=1$ with three different values of $\Omega$ in one dimension, for five different initial conditions of $\{a(z, 0), b(z, 0)\}$ for each value of the set of $\gamma, h, \Omega$. All the numerical integrations ultimately give the result $a(z, t)$ $=b(z, t)$ for the whole space. One easily confirms the existence of this particular solution. Noting this fact, we consider the particular case, $a(\boldsymbol{r}, t)=b(\boldsymbol{r}, t)$. Equations (6.33) and (6.34) are reduced to

$$
\dot{a}(\boldsymbol{r}, t)=\bar{h} a-\bar{h} a^{3}+\nabla^{2} a .
$$

For a one-dimensional system, Eq. (6.36) possesses the stationary kink and antikink solutions for the boundary condition $|a( \pm \infty)|=1$,

$$
a(z)=b(z)= \pm \tanh \left(\frac{z-z_{0}}{\xi}\right), \quad \xi=\sqrt{\frac{2}{\bar{h}}} .
$$

It should be noted that the particular solution $a(\boldsymbol{r}, t)$ $=b(\boldsymbol{r}, t)$ for any $\boldsymbol{r}$ exists only for Eqs. (6.34) and (6.35), but not for Eqs. (6.30) and (6.31).

\section{E. Domain walls between $C_{X Y B 1}$ and $C_{X Y B 3}$ in the $X Y$-SBO phase}

Finally we consider the coexistence of $C_{X Y B 1}$ and $C_{X Y B 3}$. From the symmetry of the solutions, domain walls connecting $C_{X Y B 2}$ and $C_{X Y B 4}$ can be discussed in the same way as those for $C_{X Y B 1}$ and $C_{X Y B 3}$. Let $[a(t), b(t)]$ be a quantity that takes the value $(+1,+1)[(-1,-1)]$ when the phase point is on $C_{X Y B 1}\left(C_{X Y B 3}\right)$ at time $t$. The quantities $a(t)$ and $b(t)$ are extended to the case when there exist spatial variations. The variables $a(\boldsymbol{r}, t)$ and $b(\boldsymbol{r}, t)$ defined by

$$
\begin{aligned}
& X(\boldsymbol{r}, t)=\frac{1+a(\boldsymbol{r}, t)}{2} X_{1}(t)+\frac{1-a(\boldsymbol{r}, t)}{2} X_{3}(t), \\
& Y(\boldsymbol{r}, t)=\frac{1+b(\boldsymbol{r}, t)}{2} Y_{1}(t)+\frac{1-b(\boldsymbol{r}, t)}{2} Y_{3}(t)
\end{aligned}
$$

measure how close the local order parameter $[X(\boldsymbol{r}, t), Y(\boldsymbol{r}, t)]$ is to either $C_{X Y B 1}$ or $C_{X Y B 3}$. Inserting Eqs. (6.38) and (6.39) into Eq. (2.1), after some algebra we obtain the equations the same as Eqs. (6.30), (6.31), (6.32), and (6.33) with the replacement of $X_{4}(t)$ by $X_{3}(t)$ and $Y_{4}(t)$ by $Y_{3}(t)$ in Eqs. (6.32) and (6.33).

In order to see the long-time dynamics of $a$ and $b$, we replace temporally periodic coefficients of the equations for $a$ and $b$ by their time averages. One easily finds that the equations of motion for $a$ and $b$ with the averaged coefficient again yield the particular solution $a(\boldsymbol{r}, t)=b(\boldsymbol{r}, t)$. This fact makes us consider the particular case $a(\boldsymbol{r}, t)=b(\boldsymbol{r}, t)$. In this case, we obtain the equation the same as Eq. (6.36). In a one-dimensional system with the coordinate $z$, the equation for $a$ possesses the stationary kink and antikink solutions for the boundary condition $|a( \pm \infty)|=1$ which has the same form as Eq. (6.37).

An alternative discussion on the domain walls for spin variables themselves is given in Appendix B.

\section{SUMMARY AND CONCLUDING REMARKS}

In the present paper, we discussed DPTs in the anisotropic $X Y$ spin system in a periodically oscillating magnetic field below the critical temperature $T_{c}$, using the GinzburgLandau equation with an oscillating magnetic field.

In the first half of the present paper, we studied types of spatially uniform oscillation, dropping out the $\nabla^{2} \psi$ term. After discussing the particular symmetries of the present system, we examined the stability of the SRO for different values of the amplitude $h$ and the frequency $\Omega$ of the external field. This was done by numerically calculating the largest Floquet exponent of the particular oscillation. Thus we obtained the phase diagram in the space spanned by $\Omega$ and $h$. It was found that there are at most four types of oscillations, depending on the magnitude of the anisotropy parameter $\gamma$.

Furthermore, we developed the Landau expansion of the equation of motion near several kinds of DPTs to clarify their universality classes. All the transitions seem to be the same as that of the Ising spin system in thermal equilibrium. However, since we used the Landau equation with the timeaveraged coefficients, some of the characteristic features of the DPTs might be lost. This is one of the future problems of studying explicit oscillatory characteristics of the DPTs.

In the second half of the present paper, we discussed possible forms of the domain walls connecting several types of dynamical phases in a one-dimensional system, employing the variables $a$ and $b$ to parametrize the domain structure. In 
the Ising-SBO phase with a positive anisotropy and also in the $X Y$-SRO phase for a negative anisotropy, it turned out that possible forms of the domains are described with the Néel wall and the Bloch wall known in the anisotropic $X Y$ spin system without an external field. Furthermore, the wall structure in the $X Y$-SBO phase is found to be different from the ordinary Bloch wall form. Although we reported possible types of domain walls in the present paper, their precise stability should be examined in the future.

In comparison with the DPT observed in the Ising spin systems, there are several differences in the DPTs in the anisotropic $X Y$ spin system. First, in contrast to that there is only one DPT in the Ising spin system, there can exist three kinds of DPTs, depending on the magnitude of the anisotropy parameter $\gamma$. This fact is due to the existence of four dynamical phases in the present system: Ising-SRO, Ising-SBO, $X Y$-SRO, and $X Y$-SBO. The second is about the universality class of DPTs. In a previous paper [9], we confirmed that the DPT observed in the Ising spin system belongs to the same universality class as that of the Ising spin system of conventional critical phenomena in thermodynamic equilibrium. This was shown by deriving the Landau-type effective equation of motion around the SRO near the DPT. As shown in Sec. III, however, associated with the transitions between Ising-SRO and $X Y$-SRO and also the transition between Ising-SBO and $X Y$-SBO, the number of order parameters relevant to the transition changes. The third is the possibility of the existence of the DPT above $T_{c}$. Examining the Floquet exponent of the SRO, one can prove that there exists no DPT in the Ising spin system above $T_{c}$. However, we cannot prove the nonexistence of DPTs in the anisotropic XY spin system in an oscillating field above $T_{c}$. The fourth difference is about the form of domain walls. In the Ising spin system, the domain wall is the Néel wall. On the contrary, in the anisotropic $X Y$ spin system, one observes the Bloch wall as well as the Néel wall which connect two oscillatory domains.

In the SBO phase of the Ginzburg-Landau equation in an oscillating field (1.1), the switching phenomenon between two SBO phases in the presence of thermal noise has been reported [9]. It is expected that the presence of thermal noise may cause the switching phenomena between several kinds of dynamical phases in the present system. The study on this subject is left for a future study. Furthermore, study on the ordering process beyond the DPTs in this system is an interesting problem. This is left for a future study, as well. The study of anisotropic spin system with an oscillating magnetic field is quite interesting, and is important from the viewpoint of nonlinear-nonequilibrium dynamics of spin systems. Experimental study as well as theoretical study is strongly expected to be carried out [12].

Note added in proof. Very recently, Monte Carlo studies of the dynamic phase transition in a ferromagnetic anisotropic Heisenberg spin system in an oscillating magnetic field in a thin film were carried out in [12].

\section{ACKNOWLEDGMENT}

The authors would like to thank Per A. Rikvold for discussions during his stay at Kyoto University.

\section{APPENDIX A: ASYMPTOTIC SOLUTIONS FOR A WEAK FIELD OR A HIGH FREQUENCY}

In this appendix, the perturbation expansion of asymptotic solutions for a weak field or a high frequency are briefly described.

\section{Oscillation in a weak field}

In a weak external field the dynamics shows small amplitude oscillations around the stable zero-field fixed points $X^{s s}=\left(X^{s s}, Y^{s s}\right)$. By expanding the deviations $X-X^{s s}, Y$ $-Y^{s s}$ in the power series of $h$, and by inserting these expressions into Eq. (2.5), the low-order equations in $h$ yield the perturbation equations of motion for the deviations. The results are summarized as follows.

Case A. $\gamma>0$. To the lowest order with respect to $h$, the equations of motion are solved as

$$
\begin{gathered}
\left(\begin{array}{l}
X(t) \\
Y(t)
\end{array}\right)=\left(\begin{array}{c} 
\pm \sqrt{1+\gamma}+x_{1}(t) h \\
0
\end{array}\right), \\
x_{1}(t)=\frac{1}{\sqrt{4(1+\gamma)^{2}+\Omega^{2}}} \cos \left(\Omega t-\alpha_{1}\right), \\
\tan \alpha_{1}=\frac{\Omega}{2(1+\gamma)} .
\end{gathered}
$$

Equations (A1) and (A2) have the Ising-SBO symmetry. These trajectories are compared in Fig. 11(a) with those from the original equation of motion (2.5). One finds that the dynamics under a weak field for $\gamma>0$ is well approximated by Eqs. (A1) and (A2).

Case B. $\gamma=0$. By putting $\psi=\rho e^{i \theta}, \rho$ and $\theta$ being the amplitude and the phase, respectively, and by inserting the expansion

$$
\rho=1+a_{1} h+a_{2} h^{2}+\cdots
$$

into Eq. (2.5), the lowest-order equations are determined as

$$
\begin{gathered}
\dot{\theta}(t)=-h\left(1-a_{1} h\right) \cos (\Omega t) \sin \theta, \\
\dot{a}_{1}(t)=-2 a_{1}+\cos (\Omega t) \cos \theta .
\end{gathered}
$$

The expansion coefficients $a_{2}$, etc., are successively obtained, e.g., as

$$
\dot{a}_{2}(t)=-2 a_{2}-3 a_{1}^{2} .
$$

Case C. $\gamma<0$. To the lowest order with respect to $h$, the equations of motion are solved as

$$
\begin{gathered}
\left(\begin{array}{l}
X(t) \\
Y(t)
\end{array}\right)=\left(\begin{array}{c}
x_{1}(t) h \\
\pm\left[\sqrt{1-\gamma}+y_{2}(t) h^{2}\right]
\end{array}\right), \\
x_{1}(t)=\frac{1}{\sqrt{4 \gamma^{2}+\Omega^{2}}} \cos \left(\Omega t-\alpha_{2}\right), \\
y_{2}(t)=A+B \cos \left(2 \Omega t-\alpha_{3}\right),
\end{gathered}
$$

$$
\tan \alpha_{2}=\frac{\Omega}{2 \gamma}, \quad \tan \alpha_{3}=\frac{\Omega\left(\Omega^{2}-4 \gamma\right)}{(3 \gamma+1) \Omega^{2}+4 \gamma^{2}(\gamma-1)},
$$



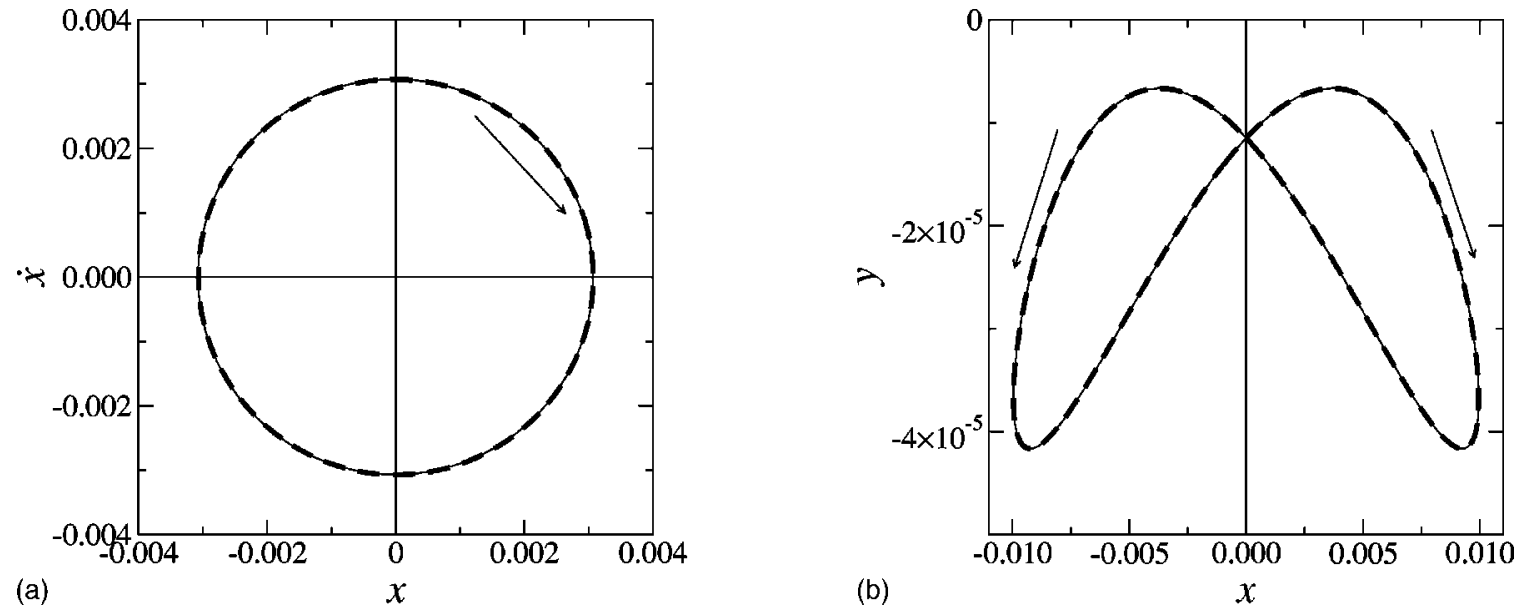

FIG. 11. Comparison of the approximate trajectories (solid lines) with the exact ones (broken lines) for a weak amplitude for $x=X$ $-X^{s s}$ and $y=Y-Y^{s s}$, where the parameter values are (a) $\gamma=0.30, h=0.01$, and $\Omega=1.0$, and (b) $\gamma=-0.05, h=0.01$, and $\Omega=1.0$. Arrows show the movement of the phase points in the course of time. In (a), one of the two symmetric Ising-SBO trajectories with $\bar{X}>0$ is plotted. In (b), one of the two symmetric $X Y$-SRO trajectories with $\bar{Y}>0$ is plotted.

$$
A=-\frac{\sqrt{1-\gamma}}{4\left(4 \gamma^{2}+\Omega^{2}\right)(1-\gamma)},
$$

$B$

$$
=\frac{\sqrt{(1-\gamma)\left\{\left[(3 \gamma+1) \Omega^{2}+4 \gamma^{2}(\gamma-1)\right]^{2}+\Omega^{2}\left(\Omega^{2}-4 \gamma\right)^{2}\right\}}}{\left[4(1-\gamma)^{2}+4 \Omega^{2}\right]\left(4 \gamma^{2}+\Omega^{2}\right)^{2}} .
$$

These equations have the $X Y$-SRO symmetry. The comparison of the trajectory obtained from Eqs. (A7), (A8), and (A9) with that from Eq. (2.5) is made in Fig. 11(b). One finds that the dynamics under a weak field for $\gamma<0$ is well approximated by Eqs. (A7), (A8), and (A9).

\section{Oscillation in a high-frequency region}

Let us consider the asymptotic dynamics for a highfrequency region. Numerical simulation shows that the spin is almost perpendicular to the applied field for a sufficiently large frequency. This is because the spin variable cannot simultaneously follow the rapid change of the applied field. Furthermore, it is observed that the Ising-SBO and the $X Y$-SRO stably exist, respectively, for $\gamma>0$ and for $\gamma \leqslant 0$ in a high-frequency field. These observations suggest the use of following expansion:

$$
\left\{\begin{array}{l}
X(t)= \pm \sqrt{1+\gamma}+x(t) \\
Y(t)=0
\end{array} \quad(\gamma>0),\right.
$$

$$
\begin{aligned}
& X(t)=x(t), \\
& Y(t)= \pm \sqrt{1-\gamma}+y(t)(\gamma \leqslant 0),
\end{aligned}
$$

with small $|x(t)|$ and $|y(t)|$. The dynamics of the perturbations $x$ and $y$ with the mode expansion are summarized as follows.

Case A. $\gamma>0$. With the mode expansion $x(t)=x_{0}(\tau)$ $+x_{1}(\tau) e^{i \tau}+x_{1}^{*}(\tau) e^{-i \tau} \quad(\tau \equiv \Omega t) \quad$ by discarding highfrequency modes, and by making use of the evaluation $x_{0}$ $=O\left(x_{1}^{2}\right)$, we obtain

$$
\begin{gathered}
\frac{d x_{0}(\tau)}{d \tau}=\epsilon\left(-2 a_{+}^{2} x_{0}-6 a_{+}\left|x_{1}\right|^{2}\right), \\
\frac{d x_{1}(\tau)}{d \tau}+i x_{1}=\epsilon\left(-2 a_{+}^{2} x_{1}-6 a_{+} x_{0} x_{1}+\frac{h}{2}\right),
\end{gathered}
$$

where $a_{+}= \pm \sqrt{1+\gamma}$ and $\epsilon \equiv \Omega^{-1}$. The steady-state solution is given as $x_{1} \approx-i h \epsilon / 2$ and $x_{0}=-3 h^{2} \epsilon^{2} / 4 a_{+}$, and therefore one obtains

$$
X(t) \approx a_{+}+\frac{h}{\Omega} \sin (\Omega t)-\frac{3}{4 a_{+}}\left(\frac{h}{\Omega}\right)^{2},
$$

up to $O\left(\Omega^{-2}\right)$, and $Y(t)=0$. One can easily prove the linear stability of the above solution. The comparison of the trajectory obtained from Eq. (A14) with that from Eq. (2.5) is made in Fig. 12(a). In the figure, from the symmetry of the Ising-SBO solutions, there exists another trajectory, which is not shown in Fig. 12(a). One finds that Eq. (A14) gives quite a good approximation for $\gamma>0$ in a high-frequency region.

Case B. $\quad \gamma \leqslant 0$. With the expansion $x(t)=x_{1}(\tau) e^{i \tau}$ $+x_{1}^{*}(\tau) e^{-i \tau}$ and $y(t)=y_{0}(\tau)+y_{2}(\tau) e^{2 i \tau}+y_{2}^{*}(\tau) e^{-2 i \tau}(\tau$ $=\Omega t$ ), we get 


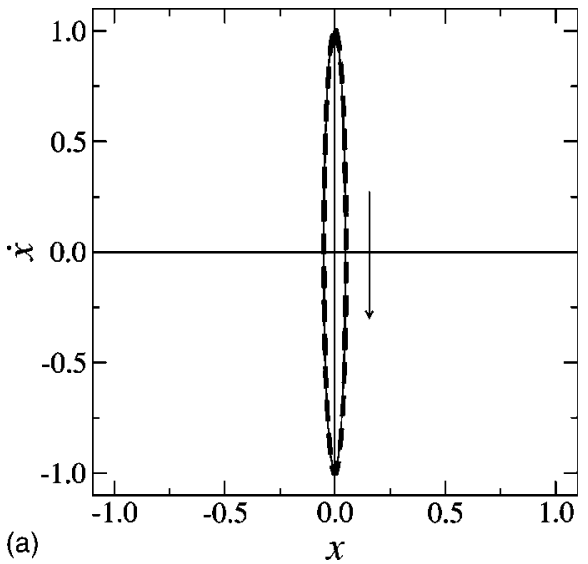

(b)

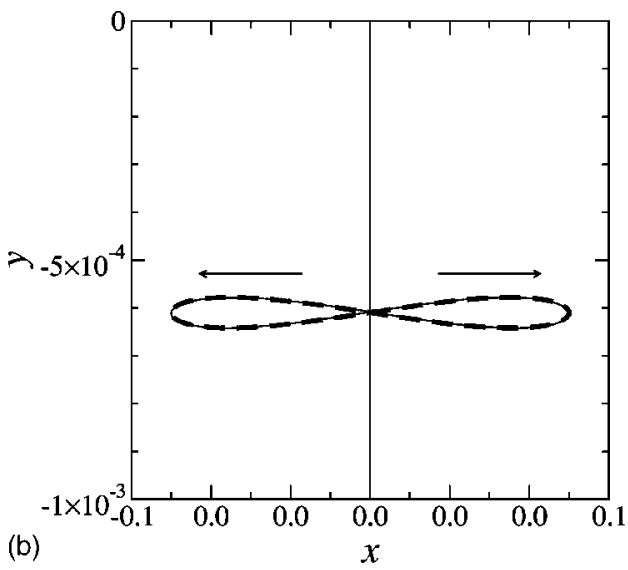

FIG. 12. Comparison of the approximate trajectories (solid lines) with the exact ones (broken lines) for a high-frequency oscillation for $x=X-X^{s s}$ and $y=Y$ $-Y^{s s}$, where the parameter values are (a) $\gamma=0.30, \quad h=1.00, \quad \Omega$ $=20.0$ and (b) $\gamma=-0.05, h$ $=1.00, \Omega=20.0$. Arrows show the movement of the phase points in the course of time.

$$
\begin{gathered}
\frac{d x_{1}(\tau)}{d \tau}+i x_{1}=\epsilon\left(-3\left|x_{1}\right|^{2} x_{1}-2 a_{-} x_{1} y_{0}\right. \\
\left.-2 a_{-} x_{1}^{*} y_{2}+2 \gamma x_{1}+\frac{h}{2}\right), \\
\frac{d y_{0}(\tau)}{d \tau}=-\epsilon\left(2 a_{-}^{2} y_{0}+2 a_{-}\left|x_{1}\right|^{2}\right), \\
\frac{d y_{2}(\tau)}{d \tau}+2 i y_{2}=-\epsilon\left(2 a_{-}^{2} y_{2}+a_{-} x_{1}^{2}\right),
\end{gathered}
$$

where $a_{-}= \pm \sqrt{1-\gamma}$ and $\epsilon=\Omega^{-1}$. Solving the steady state solution of the above equation, we obtain

$X(t)=\frac{h}{\Omega} \sin (\Omega t), \quad Y(t)=a_{-}-\frac{1}{4 a_{-}}\left(\frac{h}{\Omega}\right)^{2}+\frac{h a_{-}}{4 \Omega^{3}} \sin (2 \Omega t)$

up to $O\left(\Omega^{-3}\right)$. After a slight calculation, one can prove the linear stability of the above solution. The comparison of the trajectory obtained from Eq. (A18) with that from Eq. (2.5) is made in Fig. 12(b). From the symmetry of the $X Y$-SRO solutions, there exists another trajectory, which is not shown in Fig. 12(b). One finds that Eq. (A18) gives quite a good approximation for $\gamma \leqslant 0$ in a high-frequency region.

\section{APPENDIX B: MODE EXPANSION ANALYSIS FOR DOMAIN WALL SOLUTIONS}

Topological kinks or domain wall solutions for Eq. (2.1) in a one-dimensional system are again discussed from a slightly different viewpoint by employing the mode expansion analysis discussed in Sec. IV. In this appendix, the space coordinate is represented by $x$.

There is a possibility that the entire spatial configuration oscillates along the one-dimensional axis in response to the oscillating field. This may be taken into account by introducing the time-dependent "drift velocity" $v(t)$. Thus using the moving flame, $z=x-x_{0}(t)$, whose coordinate center is time dependent with the velocity $v(t)=\dot{x}_{0}(t)$, let us replace $\psi(x, t)$ with $\psi\left[x-x_{0}(t), t\right]$ in Eq. (2.1). Then, the time and spatial derivatives of $\psi$ are replaced as $\partial_{t} \psi \rightarrow \partial_{t} \psi-v \partial_{z} \psi$ and $\nabla \psi \rightarrow \nabla_{z} \psi$. The equations of motion for the real and imaginary components are given by

$$
\partial_{t} X-v(t) \nabla_{z} X=X-\left(X^{2}+Y^{2}\right) X+\gamma X+\nabla_{z}^{2} X+h \cos (\Omega t),
$$

$$
\partial_{t} Y-v(t) \nabla_{z} Y=Y-\left(X^{2}+Y^{2}\right) Y-\gamma Y+\nabla_{z}^{2} Y
$$

Let us expand $X$ and $Y$ in terms of Fourier components as

$$
X(z, t)=\sum_{n=-\infty}^{\infty} X_{n}(z, t) e^{i n \Omega t}, \quad Y(z, t)=\sum_{n=-\infty}^{\infty} Y_{n}(z, t) e^{i n \Omega t}
$$

where $X_{n}=X_{-n}^{*}$ and $Y_{n}=Y_{-n}^{*}$. Likewise, $v(t)$ is expanded as

$$
v(t)=\sum_{n=-\infty}^{\infty} v_{n} e^{i n \Omega t}, \quad v_{n}=v_{-n}^{*} .
$$

The substitution of these expansions into Eqs. (B1) and (B2) yields a set of equations for $X_{n}(z, t)$ and $Y_{n}(z, t)$,

$$
\begin{aligned}
\dot{X}_{n}+i n \Omega X_{n}= & \sum_{n_{1}} v_{n_{1}} \nabla_{z} X_{n-n_{1}}+(1+\gamma) X_{n} \\
& -\sum_{n_{1}, n_{2}} X_{n_{1}} X_{n_{2}} X_{n-n_{1}-n_{2}} \\
& -\sum_{n_{1}, n_{2}} Y_{n_{1}} Y_{n_{2}} X_{n-n_{1}-n_{2}}+\nabla_{z}^{2} X_{n} \\
& +\frac{h}{2}\left(\delta_{n, 1}+\delta_{n,-1}\right), \\
\dot{Y}_{n}+i n \Omega Y_{n}= & \sum_{n_{1}} v_{n_{1}} \nabla_{z} Y_{n-n_{1}}+(1-\gamma) Y_{n} \\
& -\sum_{n_{1}, n_{2}} Y_{n_{1}} Y_{n_{2}} Y_{n-n_{1}-n_{2}} \\
& -\sum_{n_{1}, n_{2}} X_{n_{1}} X_{n_{2}} Y_{n-n_{1}-n_{2}}+\nabla_{z}^{2} Y_{n} .
\end{aligned}
$$


Hereafter, we use the simplest approximation to retain only the modes $n=0, \pm 1$. As seen in Sec. IV, this set of modes is the minimum one describing DPTs. The equations of motion for $n=0$ in Eqs. (B5) and (B6) are given as

$$
\begin{aligned}
\dot{X}_{0}= & v_{0} \nabla_{z} X_{0}+v_{-1} \nabla_{z} X_{1}+v_{1} \nabla_{z} X_{-1}+\left[1+\gamma-6\left|X_{1}\right|^{2}\right. \\
& \left.-2\left|Y_{1}\right|^{2}\right] X_{0}-\left(X_{0}^{2}+Y_{0}^{2}\right) X_{0} \\
& -2\left(X_{1} Y_{-1}+X_{-1} Y_{1}\right) Y_{0}+\nabla_{z}^{2} X_{0}, \\
\dot{Y}_{0}= & v_{0} \nabla_{z} Y_{0}+v_{-1} \nabla_{z} Y_{1}+v_{1} \nabla_{z} Y_{-1}+\left[1-\gamma-6\left|Y_{1}\right|^{2}\right. \\
& \left.-2\left|X_{1}\right|^{2}\right] Y_{0}-\left(Y_{0}^{2}+X_{0}^{2}\right) Y_{0} \\
& -2\left(Y_{1} X_{-1}+Y_{-1} X_{1}\right) X_{0}+\nabla_{z}^{2} Y_{0} .
\end{aligned}
$$

Likewise, those for $n=1$ are written as

$$
\begin{aligned}
\dot{X}_{1}+i \Omega X_{1}= & v_{0} \nabla_{z} X_{1}+v_{1} \nabla_{z} X_{0}+\left[1+\gamma-3 X_{0}^{2}\right. \\
& \left.-2\left|Y_{1}\right|^{2}-Y_{0}^{2}\right] X_{1}-3\left|X_{1}\right|^{2} X_{1}-2 X_{0} Y_{0} Y_{1} \\
& -Y_{1}^{2} X_{-1}+\nabla_{z}^{2} X_{1}+\frac{h}{2}, \\
\dot{Y}_{1}+i \Omega Y_{1}= & v_{0} \nabla_{z} Y_{1}+v_{1} \nabla_{z} Y_{0}+\left[1-\gamma-3 Y_{0}^{2}\right. \\
& \left.-2\left|X_{1}\right|^{2}-X_{0}^{2}\right] Y_{1}-3\left|Y_{1}\right|^{2} Y_{1}-2 X_{0} Y_{0} X_{1} \\
& -X_{1}^{2} Y_{-1}+\nabla_{z}^{2} Y_{1} .
\end{aligned}
$$

Now we focus our attention to domain wall solutions, and regard $z$ as the one-dimensional coordinate. The boundary conditions for the sets of the field variables $\left(X_{0}, Y_{0}\right)$ and $\left(X_{1}, Y_{1}\right)$ in a single-wall configuration are given as

$$
\left(X_{0}, Y_{0}\right) \rightarrow\left(X_{0}^{ \pm}, Y_{0}^{ \pm}\right), \quad\left(X_{1}, Y_{1}\right) \rightarrow\left(X_{1}^{ \pm}, Y_{1}^{ \pm}\right),
$$

where each of the superscript \pm specifies the boundary value of $\left(X_{j}, Y_{j}\right)(j=0,1)$ at each of boundaries $z \rightarrow \pm \infty$. It is natural to impose that the boundary values $\left(X_{j}, Y_{j}\right)(j=0,1)$ belong to the uniform stationary solutions of Eqs. (B7), (B8), (B9), and (B10). Thus the boundary values are determined by

$$
\begin{aligned}
0= & {\left[1+\gamma-6\left|X_{1}^{ \pm}\right|^{2}-2\left|Y_{1}^{ \pm}\right|^{2}-\left(X_{0}^{ \pm 2}+Y_{0}^{ \pm 2}\right)\right] X_{0}^{ \pm} } \\
& -2\left(X_{1}^{ \pm} Y_{-1}^{ \pm}+X_{-1}^{ \pm} Y_{1}^{ \pm}\right) Y_{0}^{ \pm}, \\
0= & {\left[1-\gamma-6\left|Y_{1}^{ \pm}\right|^{2}-2\left|X_{1}^{ \pm}\right|^{2}-\left(Y_{0}^{ \pm 2}+X_{0}^{ \pm 2}\right)\right] Y_{0}^{ \pm} } \\
& -2\left(Y_{1}^{ \pm} X_{-1}^{ \pm}+Y_{-1}^{ \pm} X_{1}^{ \pm}\right) X_{0}^{ \pm}, \\
-\frac{h}{2}= & {\left[1+\gamma-i \Omega-3 X_{0}^{ \pm 2}-Y_{0}^{ \pm 2}-2\left|Y_{1}^{ \pm}\right|^{2}-3\left|X_{1}^{ \pm}\right|^{2}\right] X_{1}^{ \pm} } \\
& -\left(2 X_{0}^{ \pm} Y_{0}^{ \pm}+Y_{1}^{ \pm} X_{-1}^{ \pm}\right) Y_{1}^{ \pm}, \\
0= & {\left[1-\gamma-i \Omega-3 Y_{0}^{ \pm 2}-X_{0}^{ \pm 2}-2\left|X_{1}^{ \pm}\right|^{2}-3\left|Y_{1}^{ \pm}\right|^{2}\right] Y_{1}^{ \pm} } \\
- & \left(2 X_{0}^{ \pm} Y_{0}^{ \pm}+X_{1}^{ \pm} Y_{-1}^{ \pm}\right) X_{1}^{ \pm} .
\end{aligned}
$$

Here the roots for $X_{0}^{ \pm}$and $Y_{0}^{ \pm}$are degenerate, and satisfy $\left|X_{0}^{+}\right|=\left|X_{0}^{-}\right|$and $\left|Y_{0}^{+}\right|=\left|Y_{0}^{-}\right|$from the symmetry.

We use the boundary conditions $X_{0}^{-}=-X_{0}^{+}$and $Y_{0}^{ \pm}=0$ for the Ising-SBO phase, and $X_{0}^{ \pm}=0, Y_{0}^{-}=-Y_{0}^{+}$for the $X Y$-SRO phase. In addition, $Y_{1}^{ \pm}=0$ holds for both phases. Substituting $X_{0}^{ \pm} Y_{0}^{ \pm}=0$ and $Y_{1}^{ \pm}=0$ into Eq. (B14), we obtain

$$
\begin{gathered}
X_{1}^{ \pm}=-\frac{h e^{i \tan ^{-1}\left(\Omega / A^{ \pm}\right)}}{2 \sqrt{\Omega^{2}+A_{ \pm}^{2}}}, \\
A_{ \pm} \equiv 1+\gamma-3\left|X_{0}^{ \pm}\right|^{2}-Y_{0}^{ \pm 2}-3\left|X_{1}^{ \pm}\right|^{2} .
\end{gathered}
$$

Thus it is found that $X_{1}$ is uniquely determined and common at both boundaries in both Ising-SBO and $X Y$-SRO phases, i.e., $X_{1}^{+}=X_{1}^{-}$, since $X_{1}^{ \pm}$are functions of $\left|X_{0}^{ \pm}\right|$and $\left|Y_{0}^{ \pm}\right|$.

The boundary conditions for the $X Y$-SBO phases are somewhat complicated, since $Y_{1}^{ \pm} \neq 0$ and $X_{0}^{ \pm} Y_{0}^{ \pm} \neq 0$. We have a possibility to use the following three different boundary conditions for $X_{0}$ and $Y_{0}$ :

$$
\begin{aligned}
& \text { (i) }\left(X_{0}^{-}, Y_{0}^{-}\right)=\left(X_{0}^{+},-Y_{0}^{+}\right) \text {, } \\
& \text { (ii) }\left(X_{0}^{-}, Y_{0}^{-}\right)=\left(-X_{0}^{+}, Y_{0}^{+}\right) \text {, } \\
& \text { (iii) }\left(X_{0}^{-}, Y_{0}^{-}\right)=\left(-X_{0}^{+},-Y_{0}^{+}\right) \text {. }
\end{aligned}
$$

It is natural to require that the boundary condition for $X_{1}^{ \pm}$is the same at both boundaries, i.e., $X_{1}^{+}=X_{1}^{-}$, as a physically preferable state. For case (i), from Eqs. (B14) and (B15), to take the boundary condition for $Y_{1}$ so as to have the same parity as $Y_{0}$, i.e., $Y_{1}^{+}=\left(Y_{0}^{-} / Y_{0}^{+}\right) Y_{1}^{-}$, allows us to set the condition $X_{1}^{+}=X_{1}^{-}$. Similarly, for case (ii), to take the boundary condition for $Y_{1}$ so as to have the same parity as $X_{0}$, i.e., $Y_{1}^{+}=\left(X_{0}^{-} / X_{0}^{+}\right) Y_{1}^{-}$, allows us to set the condition $X_{1}^{+}=X_{1}^{-}$. For case (iii), the simplest boundary conditions for $X_{1}$ and $Y_{1}$ are $X_{1}^{+}=X_{1}^{-}$and $Y_{1}^{+}=Y_{1}^{-}$. Therefore, the conditions $Y_{1}^{+}=\left(X_{0}^{-} Y_{0}^{-} / X_{0}^{+} Y_{0}^{+}\right) Y_{1}^{-}$and $X_{1}^{+}=X_{1}^{-}$satisfy all the cases including $Y_{1}^{ \pm}=0$. This also indicates that $Y_{1}$ necessarily has a kink structure when either $X_{0}$ or $Y_{0}$ has a domain wall structure in the $X Y$-SBO phase.

In the Ising-SBO and $X Y$-SRO phases, the lowest-order variables for describing a domain wall configuration are $X_{0}$ and $Y_{0}$, while, in the $X Y$-SBO phase, $Y_{1}$ also participates with them for the boundary conditions (i) and (ii). In the following, we will deal with the domain wall solutions for the Ising-SBO phase, $X Y$-SRO phase, and also with the case for the boundary condition (iii) in the $X Y$-SBO phase. For these situations, the domain wall solutions for $X_{0}$ and $Y_{0}$ are approximated by regarding $X_{1}$ and $Y_{1}$ as uniform in Eqs. (B7) and (B8). Substituting $X_{1}=X_{1}^{+}$and $Y_{1}=Y_{1}^{+}\left(=Y_{1}^{-}\right)$ into Eqs. (B7) and (B8), the equation determining the domain wall solution for $\psi_{0}=X_{0}+i Y_{0}$ is written as

$$
\begin{gathered}
-v_{0} \partial_{z} \psi_{0}=c_{0} \psi_{0}-\left|\psi_{0}\right|^{2} \psi_{0}+\gamma_{0} \psi_{0}^{*}+\partial_{z}^{2} \psi_{0}, \\
c_{0}=1-4\left|X_{1}^{+}\right|^{2}-4\left|Y_{1}^{+}\right|^{2},
\end{gathered}
$$




$$
\gamma_{0}=\gamma-2\left|X_{1}^{+}\right|^{2}+2\left|Y_{1}^{+}\right|^{2}-2 i\left(X_{1}^{+} Y_{-1}^{+}+X_{-1}^{+} Y_{1}^{+}\right),
$$

where the boundary values $\left[X_{0}(z= \pm \infty), Y_{0}(z= \pm \infty)\right]=$ $\left( \pm X_{0}^{+}, \pm Y_{0}^{+}\right)$and $\left(X_{1}^{+}, Y_{1}^{+}\right)$must satisfy Eqs. (B14) and (B15). In Eq. (B20) the effective anisotropy parameter $\gamma_{0}$ is complex if $Y_{1}^{+} \neq 0$, whose phase defined by $\gamma_{0}=\left|\gamma_{0}\right| e^{2 i \theta_{0}}$ is determined as

$$
\tan 2 \theta_{0}=-2 \frac{X_{1}^{+} Y_{-1}^{+}+X_{-1}^{+} Y_{1}^{+}}{\gamma-2\left|X_{1}^{+}\right|^{2}+2\left|Y_{1}^{+}\right|^{2}} .
$$

Since for one value of $Y_{1}^{+}$there is another degenerate value $-Y_{1}^{+}$, the phase $\theta_{0}$ has two opposite values.

By replacing the phase of $\psi_{0}$ as $\psi_{0}=\widetilde{\psi}_{0} e^{i \theta_{0}}$, we hereafter solve Eq. (B18) for $\widetilde{\psi}_{0}$. In order to determine $v_{0}$, multiplying Eq. (B18) by $\partial_{z} \widetilde{\psi}_{0}^{*}$ and adding its complex conjugate to it, we get

$$
-2 v_{0}\left|\partial_{z} \widetilde{\psi}_{0}\right|^{2}=\partial_{z} \mu(z)
$$

where

$$
\mu(z)=c_{0}\left|\widetilde{\psi}_{0}\right|^{2}-\frac{1}{2}\left|\widetilde{\psi}_{0}\right|^{4}+\frac{1}{2}\left|\gamma_{0}\right|\left(\widetilde{\psi}_{0}^{* 2}+\widetilde{\psi}_{0}^{2}\right)+\left|\partial_{z} \widetilde{\psi}_{0}\right|^{2} .
$$

By integrating both sides from $z=-\infty$ to $\infty, v_{0}$ is determined as

$$
v_{0}=-\frac{1}{2 \sigma}[\mu(\infty)-\mu(-\infty)], \quad \sigma \equiv \int_{-\infty}^{\infty} d z\left|\partial_{z} \widetilde{\psi}_{0}\right|^{2} .
$$

Here the condition $\widetilde{\psi}_{0}(z=-\infty)=-\widetilde{\psi}_{0}(z=\infty)$ leads to $\mu(\infty)=\mu(-\infty)$, and therefore we get $v_{0}=0$. Equation (B18), using $v_{0}=0$, yields domain wall solutions when $c_{0}$ $>0$, which turn out to have the following two types of solutions:

$$
\begin{array}{cc}
\widetilde{\psi}_{0}^{I}(z)=\sqrt{c_{0}+\left|\gamma_{0}\right|} \tanh \left[\sqrt{\left(c_{0}+\left|\gamma_{0}\right|\right) / 2} z\right] & \left(\left|\gamma_{0}\right|>c_{0} / 3\right), \\
\widetilde{\psi}_{0}^{B}(z)=X_{B} \tanh \left(z / \xi_{B}\right) \pm i Y_{B} \operatorname{sech}\left(z / \xi_{B}\right) & \left(0<\left|\gamma_{0}\right|<c_{0} / 3\right),
\end{array}
$$

where $X_{B}=\sqrt{c_{0}+\left|\gamma_{0}\right|}, Y_{B}=\sqrt{c_{0}-3\left|\gamma_{0}\right|}, \xi_{B}=1 / \sqrt{2\left|\gamma_{0}\right|}$, and the corresponding solutions for $\psi_{0}$ are obtained as $\psi_{0}$ $=\widetilde{\psi}_{0} e^{i \theta_{0}}$.

We can perturbatively get the solution by incorporating the spatial variation of $X_{1}$ and $Y_{1}$. Namely, denoting the solutions obtained above as $\psi_{0}^{(0)}$ and $\psi_{1}^{(0)}$, we can expand $\psi_{0}$ and $\psi_{1}$ as

$$
\begin{aligned}
& \psi_{0}=\psi_{0}^{(0)}+\epsilon \psi_{0}^{(1)}+\epsilon^{2} \psi_{0}^{(2)}+\cdots, \\
& \psi_{1}=\psi_{1}^{(0)}+\epsilon \psi_{1}^{(1)}+\epsilon^{2} \psi_{1}^{(2)}+\cdots, \\
& v_{0}=v_{0}^{(0)}+\epsilon v_{0}^{(1)}+\epsilon^{2} v_{0}^{(2)}+\cdots, \\
& v_{1}=v_{1}^{(0)}+\epsilon v_{1}^{(1)}+\epsilon^{2} v_{1}^{(2)}+\cdots,
\end{aligned}
$$

with a certain smallness parameter $\epsilon$, and then calculate quantities $\psi_{j}^{l}, v_{j}^{l}(l=1,2, \ldots)$ order by order in power of $\epsilon$. For the Ising-SBO and $X Y$-SRO phases, $v_{1}^{(0)}$ vanishes in the lowest-order approximation, since we assumed that $X_{1}$ and $Y_{1}$ are uniform. For that case the series expansion for $v_{1}$ begins with the order $\epsilon$.
[1] T. Tomé, and M.J. de Oliveira, Phys. Rev. A 41, 4251 (1990).

[2] J.F.F. Menders, and E.J.S. Large, J. Stat. Phys. 64, 653 (1991).

[3] M. Acharyya, Phys. Rev. E 56, 2407 (1997).

[4] S.W. Sides, P.A. Rikvold, and M.A. Novotny, Phys. Rev. Lett. 81, 834 (1998).

[5] S.W. Sides, P.A. Rikvold, and M.A. Novotny, Phys. Rev. E 59, 2710 (1999).

[6] P.A. Rikvold et al., in Computer Simulation Studies in Condensed Matter Physics XIII, edited by D. P. Landau, S. P. Lewis, and H.-B. Schuttler, (Springer, Berlin, 2000), pp. 105119.

[7] G. Korniss, C.J. White, P.A. Rikvold, and M.A. Novotny,
Phys. Rev. E 63, 016120 (2001).

[8] G. Grinstein, C. Jayaprakash, and Y. He, Phys. Rev. Lett. 55, 2527 (1985).

[9] H. Fujisaka, H. Tutu, and P.A. Rikvold, Phys. Rev. E 63, 036109 (2001).

[10] M. Yamamoto, Master thesis, Graduate School of Informatics, Kyoto University, 2001.

[11] J. Guckenheimer and P. Holmes, Nonlinear Oscillations, Dynamical Systems, and Bifurcations of Vector Fields (SpringerVerlag, Berlin, 1983).

[12] H. Jang and M.J. Grimson, Phys. Rev. E 63, 066119 (2001); H. Jang, M.J. Grimson, and C.K. Hall, e-print cond-mat/0205070. 